\title{
HS in flat spacetime: the effective action method
}

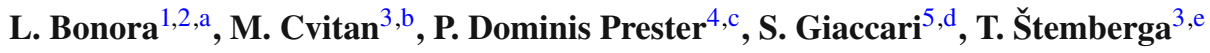 \\ ${ }^{1}$ International School for Advanced Studies (SISSA), Via Bonomea 265, 34136 Trieste, Italy \\ ${ }^{2}$ INFN, Sezione di Trieste, Trieste, Italy \\ ${ }^{3}$ Department of Physics, Faculty of Science, University of Zagreb, Bijenička cesta 32, 10000 Zagreb, Croatia \\ ${ }^{4}$ Department of Physics, University of Rijeka, Radmile Matejčić 2, 51000 Rijeka, Croatia \\ ${ }^{5}$ Department of Sciences, Holon Institute of Technology (HIT), 52 Golomb St., 5810201 Holon, Israel
}

Received: 20 November 2018 / Accepted: 7 February 2019 / Published online: 21 March 2019

(C) The Author(s) 2019

\begin{abstract}
This is the first paper in a series of three dealing with HS theories in flat spacetime. It is divided in three parts. The first part is an elaboration on the method of effective action, initiated in a previous paper. We study the properties of correlators of currents in the free fermion coupled to external higher spin (HS) potentials, and develop techniques for their explicit calculation. In particular we show how they can be calculated via ordinary Feynman diagram techniques. We also introduce the concept of curved $L_{\infty}$ algebra and show how it can be realized in the context of the fermion model. In part II we compare the results of the scalar model and those of the fermion model (coupled to HS fields). We show that the HS field formulation coming from the scalar model is the 'square' of the one ensuing from the fermion model. Finally, in part III, we analyse the possible obstructions that one may meet in constructing the effective action: these are the analogues of anomalies in ordinary gauge theories. We provide explicit and compact formulas of the latter.
\end{abstract}

\section{Introduction}

This paper and the following ones are devoted to the construction and analysis of massless field models with infinite many fields of increasing spins in flat spacetime. The construction of interacting relativistic quantum field theories with fundamental fields/particles of spin higher then two (higher spin, or HS, for short) in a flat background is a long-standing problem of theoretical physics. On the one hand there are theoretical motivations for searching such theories. On the wake of

\footnotetext{
a e-mail: bonora@sissa.it

be-mail: mcvitan@phy.hr

c e-mail: pprester@phy.uniri.hr

de-mail: stefanog@hit.ac.il

e e-mail: tstember@phy.hr
}

(super)string theories, it has been known that infinite towers of higher spin fields can soften the UV behaviour in a drastic way. The AdS/CFT correspondence offers another example in which the description of an ordinary conformal field theory can be improved by the use of a dual string theory. There are also arguments suggesting that when gravity is involved an infinite number of fields with increasing spin is necessary in order to avoid conflicts with causality [1].

On the other hand, there are some dissuasive elements in the story. Even if we ignore the experimental evidence against the existence of elementary HS particles, there seem to be theoretical obstructions: there are in the literature several no-go theorems prohibiting, under some rather standard assumptions, the existence of massless HS particles in a flat spacetime. It is not strange then that the only HS theories in $d \geq 4$ dimensions constructed so far are in the (A)dS background, through the Vasiliev program [2-5]. Studies of the massless HS theories in flat spacetime clearly suggest that such theories must violate some of the usual QFT assumptions, which are assumed in no-go theorems: either locality (see, in particular [6-9]), or minimal coupling to gravity, or finiteness of the number of particles with the mass below any finite energy scale (infinite tower of HS fields are necessary, see [10]).

We know that in the case of free HS theories, [11,12], the constructions using Wigner and Bargmann-Wigner methods goes the same way as for lower spin fields/particles, and that free field theories contain infinite towers of conserved currents of all spins. These two properties are normally starting points in constructing theories with local symmetries and their interactions with matter fields, well known examples being Maxwell/Yang-Mills gauge field theories and GR. Understandably this is the reason why most attempts to construct HS theories have moved along these same lines. Here, though, we would like to pursue another interesting avenue for exploring HS theories, opened by papers [13-15]. 
In $[13,14]$ it was shown that the Klein-Gordon field (the matter field) linearly coupled to the infinite tower of HS background potentials represented by symmetric tensor fields (metric-like formalism) $h_{(s)}^{\mu_{1} \ldots \mu_{s}}(x)$

$S[\varphi, h]=S_{0}[\varphi]+\sum_{s=0}^{\infty} \int d^{d} x \frac{1}{s !} J_{\mu_{1} \ldots \mu_{s}}^{(s)}(x) h_{(s)}^{\mu_{1} \ldots \mu_{s}}(x)$

where $S_{0}[\varphi]$ is the action for the free (massive) KG field and $J_{\mu_{1} \ldots \mu_{s}}^{(s)}$ is the spin-s current which is conserved in the free KG theory, is symmetric under local HS transformations. If one chooses for the currents the so-called simple form

$J_{\mu_{1} \ldots \mu_{s}}^{(s)}(x)=\frac{i^{s-2}}{2^{s}} \varphi(x)^{*} \stackrel{\leftrightarrow}{\partial}_{\mu_{1}} \ldots \stackrel{\leftrightarrow}{\partial}_{\mu_{s}} \varphi(x)$

then the HS symmetry has also a particularly simple form when the HS potentials are represented in a $2 d$ dimensional phase space $(x, u) .{ }^{1}$ In this case the HS gauge transformation of the HS master field potential ${ }^{2}$ is

$\delta_{\eta} h(x, u)=\left(u \cdot \partial_{x}\right) \eta(x, u)-\frac{i}{2}[h(x, u) \stackrel{\star}{,} \eta(x, u)]$

where $\star$ is the Moyal product and $\left[,^{\star}\right]$ is the commutator defined by the Moyal product. The HS master field potential $h(x, u)$ is defined by the Taylor expansion around $u=0$

$h(x, u)=\sum_{s=0}^{\infty} \frac{1}{s !} h_{(s)}^{\mu_{1} \cdots \mu_{s}}(x) u_{\mu_{1}} \cdots u_{\mu_{s}}$

and $\eta(x, u)$ is an arbitrary phase space parameter regular around $u=0$

$\eta(x, u)=\sum_{s=1}^{\infty} \frac{1}{(s-1) !} \eta^{\mu_{1} \cdots \mu_{s-1}}(x) u_{\mu_{1}} \cdots u_{\mu_{s-1}}$

where $\eta^{\mu_{1} \cdots \mu_{s-1}}(x)$ are arbitrary spacetime tensor fields parametrizing local HS transformations. By using (4) and (5) in (3) one gets that HS transformations of spacetime HS fields are of the form

$\delta_{\eta(x, u)} h_{(s)}^{\mu_{1} \cdots \mu_{s}}(x)=s \partial^{\left(\mu_{1}\right.} \eta^{\left.\mu_{2} \cdots \mu_{s}\right)}(x)+$ (non-linear terms).

The linear part has the standard form of linearised unrestricted HS transformations for $s \geq 1$.

\footnotetext{
1 Throughout the paper the position in the phase space are denoted by couples of letters $(x, u),(y, v),(z, t),(w, r)$, the first letter refers to the space-time coordinate and the second the worldline particle momentum. The letters $k, p, q$ will be reserved to the momenta of the (Fouriertransformed) physical amplitudes.

${ }^{2}$ In order to distinguish them from ordinary spacetime fields, at times we will refer to fields like $h(x, u)$ and $J(x, u)$, and $h_{a}(x, u)$ and $J_{a}(x, u)$ below, as master fields.
}

As was observed in $[13,14]$ the HS gauge transformation (3) obeys Lie-algebra type commutator ${ }^{3}$

$\left[\delta_{\eta_{1}}, \delta_{\eta_{2}}\right]=\delta_{i\left[\eta_{1}, \eta_{2}\right]}$.

While there is simplicity and elegance in the relations (3) and (7) and obvious similarity with the analogous Yang-Mills transformations, there are also some annoying aspects. First of all, we know already from electrodynamics (pure spin1 example) that coupling of $\mathrm{KG}$ field to spin-1 gauge field contains also quadratic (seagull) terms in the action, which are not present in (1). For this reason it was assumed in [13] that (1) is just a linearization, and that a consistently coupled theory contains also non-linear couplings. If so, this could in principle change the expression for HS transformations and the Lie-structure formula (7). Moreover, the necessity of a spin-0 field in the tower of HS fields, which couples to the mass term $\varphi^{*} \varphi$ (not a conserved current in the standard sense), at first sight may look strange. However, it was noted in [14], that the form of the HS transformations suggests that the spin-0 HS field should be treated as a composite field, which means that it should provide non-linear couplings of HS fields with spin $s \geq 1$ with matter. Anyway, this compositeness drastically complicates the HS structure.

Recently, in [15] it was shown that the symmetries observed in $[13,14]$ extend to other matter fields, in particular to the Dirac field $\psi$ linearly coupled to the infinite tower of HS background fields $h_{(s)}^{a \mu_{1} \ldots \mu_{s-1}}(x)$

$S[\psi, h]=S_{0}[\psi]+\sum_{s=1}^{\infty} \int d^{d} x J_{a \mu_{1} \ldots \mu_{s-1}}^{(s)}(x) h_{(s)}^{a \mu_{1} \ldots \mu_{s-1}}(x)$

where $S_{0}[\psi]$ is the action for the free Dirac field, and $J_{a \mu_{1} \ldots \mu_{s-1}}^{(s)}(x)$ is the spin-s current which is conserved for the free Dirac field. If we take a simple form for the matter currents

$$
J_{a \mu_{1} \ldots \mu_{s-1}}^{(s)}(x)=\frac{(-i)^{s-1}}{2^{s-1}(s-1) !} \bar{\psi}(x) \gamma_{a} \stackrel{\leftrightarrow}{\partial}_{\mu_{1}} \ldots \stackrel{\leftrightarrow}{\partial}_{\mu_{s-1}} \psi(x)
$$

then the action (8) is invariant under the HS gauge transformations defined by the variations

$\delta_{\varepsilon} h_{a}(x, u)=\partial_{a}^{x} \varepsilon(x, u)-i\left[h_{a}(x, u)^{\star}, \varepsilon(x, u)\right]$

where now

$h_{a}(x, u)=\sum_{s=1}^{\infty} \frac{1}{(s-1) !} h_{a}^{(s) a \mu_{1} \cdots \mu_{s-1}}(x) u_{\mu_{1}} \cdots u_{\mu_{s-1}}$.

\footnotetext{
${ }^{3}$ Using different forms for the conserved currents is equivalent to field redefinitions, which would change the form of HS transformation (3). Still, the Lie-algebra structure (7) would of course be the same.
} 
The HS transformations of spacetime HS potential fields has the form

$\delta_{\varepsilon} h_{a}^{(s) \mu_{1} \cdots \mu_{s-1}}(x)=\partial_{a} \varepsilon_{(s)}^{\mu_{1} \cdots \mu_{s-1}}(x)+$ (non-linear terms).

Several things deserve to be emphasized: (1) The HS field is different from the one coupled to the $\mathrm{KG}$ field (because HS transformations are different). (2) The form of the HS variation (10) suggests that the coupled spacetime HS fields are symmetric only in $(s-1)$ indices, one index (denoted by latin letter) standing out. It is usual to call such representations for HS fields frame-like. (3) There is no spin-0 field present, which implies that the coupling is purely linear. (4) The HS transformation (10) respects (7), with $\eta(x, u)$ substituted with $\varepsilon(x, u)$.

Our idea here is to take the universality of the HS structure as a sign that the HS symmetries obtained by linearly coupling HS fields to matter may be exact. This assumption is supported by an observation from [15] that actions for HS fields invariant under HS transformation (10) have an $L_{\infty}$ symmetry, ${ }^{4}$ a property shared by many consistent theories. In the following we will take (10) as an exact HS gauge transformation, and the frame-like approach as a starting point of our construction. We will show that (3) is related to a metric-like formulation, the latter is a composite version of the framelike one, thus clarifying the question of the multiplicity of HS field representations.

It may be surprising that we do not impose any constraint on the HS potential and gauge parameters. Simple and double traceless conditions are related to Fronsdal's approach to HS. These are conditions to be imposed, for instance, on the gauge parameters in order to guarantee the gauge invariance of the linearized HS equations of motion. As was shown by Francia and Sagnotti [6-9], these restrictions can be released at the price of allowing for a mild non-locality (mild because it involves non-physical degrees of freedom). In Refs. [33,34] we have shown on several examples that this trading between tracelessness conditions and non-locality is naturally realized in the effective actions obtained by integrating out matter fields. In [42] we show that such restrictions may be unnecessary in order to construct fully invariant (perturbatively) local HS theories.

For the sake of practicality we split our exposition in three papers. The first, this paper, is tied to the idea of effective action. We improve the analysis initiated in [15], by studying the properties of correlators of currents in the fermion model and develop techniques for their explicit calculation. In particular we show how they can be calculated via Feynman diagram techniques. We also introduce the concept of curved $L_{\infty}$ algebra and show how it can be realized in the

${ }^{4}$ For $L_{\infty}$ see $[16-25,27]$. context of the fermion model. Then we tackle the problem of the relation between the outputs of the scalar model and those of the fermion model, and show that it can be easily clarified with the idea of compositeness: the HS field formulation coming from the scalar model is the 'square' of the one ensuing from the fermion model, much as the metric is the square of the vielbein. Finally we analyse the possible obstructions that one may meet in constructing the effective action: these are the analogues of anomalies in ordinary gauge theories. The frame-like formalism allows us to provide explicit and compact formulas of the latter.

The effective action method is based on the early work of Sakharov, [28-31], revisited recently by [32-34]. The approach we use is based on the worldline quantization method, see [35-41] and others. As mentioned above here we follow in particular the line of [13-15].

The results in this paper are general, they are obtained abstracting from the idea of a locality: an effective field action is mostly nonlocal. In the follow-up of the present paper, denoted II [42], we will grow bolder. We will abandon small scale cabotage around the idea of effective action and launch ourselves in a new enterprise: instead of trying to derive explicit actions by integrating out (scalar or fermion) matter fields, we will use the wisdom (formulas and constructs) acquired in this and previous papers to integrate $L_{\infty}$, that is to determine classical (perturbatively) local theories which automatically satisfy the $L_{\infty}$ relations and in particular enjoy the HS gauge invariance/covariance. In II we will exhibit explicit examples of HS Chern-Simons and HS Yang-Mills theories, both Abelian and non-Abelian. As HS YM-like theories contain spin-2 field, they may pretend to be descriptions of gravity. In the third paper (III) we shall develop a HS geometry-like formalism, which is more suitable for a background independent framework, and use it to analyse the spin-2 sector.

\section{Part I. Functional equations and methods}

In this first part of the paper we resume the analysis started in [15] and develop it in various directions. We first summarize facts about the fermionic matter model and the relevant effective action. We then complete the analysis of the $L_{\infty}$ structure of the latter by considering the case of the curved $L_{\infty}$ algebra. We then proceed to some sample calculations of 1- and 2-point correlator and then show the connection of the perturbative method outlined in [15] with the more traditional perturbative approach based on Feynman diagrams.

\section{The method of effective action}

The fermionic matter model is

$S_{\text {matter }}=\int d^{d} x \bar{\psi}(i \gamma \cdot \partial-m) \psi$ 


$$
\begin{aligned}
& +\sum_{s=1}^{\infty} \int d^{d} x J_{a \mu_{1} \ldots \mu_{s-1}}^{(s)}(x) h_{(s)}^{a \mu_{1} \ldots \mu_{s-1}}(x) \\
= & S_{0}+S_{\text {int }} .
\end{aligned}
$$

The interaction part $S_{i n t}$ can be written in various ways

$S_{i n t}=\left\langle\left\langle J_{a}, h^{a}\right\rangle\right\rangle=\int d^{d} x \frac{d^{d} u}{(2 \pi)^{d}} J_{a}(x, u) h^{a}(x, u)$.

The (external) gauge fields are collectively represented by

$h^{a}(x, u)=\sum_{s=1}^{\infty} \frac{1}{(s-1) !} h_{(s)}^{a \mu_{1} \ldots \mu_{s-1}}(x) u_{\mu_{1}} \ldots u_{\mu_{s-1}}$,

and

$$
\begin{aligned}
J_{a}(x, u) & \delta S_{i n t} \\
= & \frac{\delta h^{a}(x, u)}{\delta} d^{d} z e^{i z \cdot u} \bar{\psi}\left(x+\frac{z}{2}\right) \gamma_{a} \psi\left(x-\frac{z}{2}\right) \\
& =\sum_{n, m=0}^{\infty} \frac{(-i)^{n} i^{m}}{2^{n+m} n ! m !} \partial^{n} \bar{\psi}(x) \gamma_{a} \partial^{m} \psi(x) \frac{\partial^{n+m}}{\partial u^{n+m}} \delta(u) \\
& =\sum_{s=1}^{\infty}(-1)^{s-1} J_{a \mu_{1} \ldots \mu_{s-1}}^{(s)}(x) \frac{\partial^{s-1}}{\partial u_{\mu_{1}} \ldots \partial u_{\mu_{s-1}}} \delta(u)
\end{aligned}
$$

which is obtained by expanding $e^{i u \cdot z}$. In order to extract $J_{a v_{1} \ldots u_{s-1}}^{(s)}(x)$ from $J_{a}(x, u)$ one must multiply it by $u_{\nu_{1}} \ldots$ $u_{v_{s-1}}$, integrate over $u$ and divide by $(s-1)$ !. Also

$$
\begin{aligned}
J_{a \mu_{1} \ldots \mu_{s-1}}^{(s)}(x)= & \frac{i^{s-1}}{(s-1) !} \frac{\partial}{\partial z^{\left(\mu_{1}\right.}} \\
& \left.\ldots \frac{\partial}{\partial z^{\left.\mu_{s-1}\right)}} \bar{\psi}\left(x+\frac{z}{2}\right) \gamma_{a} \psi\left(x-\frac{z}{2}\right)\right|_{z=0} .
\end{aligned}
$$

The gauge transformation of $h_{a}$ is ${ }^{5}$

$$
\begin{aligned}
\delta_{\varepsilon} h_{a}(x, u) & =\partial_{a}^{x} \varepsilon(x, u)-i\left[h_{a}(x, u) *{ }^{*} \varepsilon(x, u)\right] \\
& \equiv \mathcal{D}_{a}^{* x} \varepsilon(x, u),
\end{aligned}
$$

where we have introduced the covariant derivative $\mathcal{D}_{a}^{* x}=\partial_{a}^{x}-i\left[h_{a}(x, u) \stackrel{*}{,}\right]$.

The effective action is denoted $\mathcal{W}[h]$ and takes the form

$$
\begin{aligned}
\mathcal{W}[h]= & \mathcal{W}[0]+\sum_{n=1}^{\infty} \frac{1}{n !} \int \prod_{i=1}^{n} d^{d} x_{i} \frac{d^{d} u_{i}}{(2 \pi)^{d}} \mathcal{W}_{a_{1} \ldots a_{n}}^{(n)} \\
& \times\left(x_{1}, u_{1}, \ldots, x_{n}, u_{n}\right) h^{a_{1}}\left(x_{1}, u_{1}\right) \ldots h^{a_{n}}\left(x_{n}, u_{n}\right) \\
= & \mathcal{W}[0]+\sum_{n=1}^{\infty} \frac{i^{n-1}}{n !} \int \prod_{i=1}^{n} d^{d} x_{i} \frac{d^{d} u_{i}}{(2 \pi)^{d}} \\
& \times\left\langle J_{a_{1}}\left(x_{1}, u_{1}\right) \ldots J_{a_{n}}\left(x_{n}, u_{n}\right)\right\rangle h^{a_{1}}\left(x_{1}, u_{1}\right) \ldots h^{a_{n}}\left(x_{n}, u_{n}\right)
\end{aligned}
$$

\footnotetext{
5 We use the notation $h_{a}$ instead of $h_{\mu}$ as in [15] to anticipate and stress the frame-like interpretation of the master field. See further on.
}

where

$$
\begin{aligned}
& \mathcal{W}_{a_{1} \ldots a_{n}}^{(n)}\left(x_{1}, u_{1}, \ldots, x_{n}, u_{n}\right) \\
&=i^{n-1}\left\langle J_{a_{1}}\left(x_{1}, u_{1}\right) \cdots J_{a_{n}}\left(x_{n}, u_{n}\right)\right\rangle \\
&=\left(\sum_{s_{1}=1}^{\infty} \frac{\partial}{\partial u_{1 a_{1}^{(1)}}} \cdots \frac{\partial}{\partial u_{1 a_{s_{1}-1}^{(1)}}} \delta\left(u_{1}\right)\right) \cdots \\
&\left(\sum_{s_{n}=1}^{\infty} \frac{\partial}{\partial u_{n a_{1}^{(n)}}} \cdots \frac{\partial}{\partial u_{n a_{s_{n}-1}^{(n)}}} \delta\left(u_{n}\right)\right) \\
& \times\left\langle J_{a_{1} a_{1}^{(1)} \cdots a_{s_{1}-1}^{(1)}}^{\left(s_{1}\right)} \cdots J_{a_{n} a_{1}^{\left(s_{n}\right)}\left(x_{1}\right) \cdots a_{s_{n}-1}^{(n)}}^{\left(s_{n}\right)}\right\rangle .
\end{aligned}
$$

In the sequel we will assume $\mathcal{W}[0]=0$, see Appendix B.1 for a justification.

The statement of invariance under (18) is the global Ward identity (WI)

$\delta_{\varepsilon} \mathcal{W}[h]=0$.

Taking the variation with respect to $\varepsilon(x, u)$ this becomes

$$
\begin{aligned}
\sum_{n=1}^{\infty} & \frac{1}{n !} \int \prod_{i=1}^{n} d^{d} x_{i} \frac{d^{d} u_{i}}{(2 \pi)^{d}} \mathcal{D}_{x}^{* a} \mathcal{W}_{a a_{1} \ldots, a_{n}}^{(n+1)} \\
& \times\left(x, u, x_{1}, u_{1} \ldots, x_{n}, u_{n}\right) h^{a_{1}}\left(x_{1}, u_{1}\right) \ldots h^{a_{n}}\left(x_{n}, u_{n}\right)=0 .
\end{aligned}
$$

This must be true order by order in $h$, i.e.

$$
\begin{aligned}
0= & \int \prod_{i=1}^{n} d^{d} x_{i} \frac{d^{d} u_{i}}{(2 \pi)^{d}} \partial_{x}^{a} \mathcal{W}_{a a_{1} \ldots a_{n}}^{(n+1)}\left(x, u, x_{1}, u_{1} \ldots, x_{n}, u_{n}\right) \\
& \times h^{a_{1}}\left(x_{1}, u_{1}\right) \ldots h^{a_{n}}\left(x_{n}, u_{n}\right) \\
& -i n \int \prod_{i=1}^{n-1} d^{d} x_{i} \frac{d^{d} u_{i}}{(2 \pi)^{d}}\left[h^{a}(x, u) * \mathcal{W}_{a a_{1} \ldots a_{n-1}}^{(n)}\right. \\
& \left.\times\left(x, u, x_{1}, u_{1} \ldots, x_{n-1}, u_{n-1}\right)\right] \\
& \times h^{a_{1}}\left(x_{1}, u_{1}\right) \ldots h^{a_{n-1}}\left(x_{n-1}, u_{n-1}\right) .
\end{aligned}
$$

Assuming $\mathcal{W}_{\mu}^{(1)}=0$, by functionally differentiating w.r.t. $h^{b}(y, v)$ and setting $h=0$, for $n=1$ this becomes

$0=\partial_{x}^{a} \mathcal{W}_{a b}^{(2)}(x, u ; y, v)$.

Functionally differentiating also w.r.t. $h^{c}(z, t)$ (and setting $h=0$ ) we get

$$
\begin{aligned}
0= & \frac{1}{(2 \pi)^{d}}\left(\partial_{z}^{c} \mathcal{W}_{a b c}^{(3)}(x, u ; y, v ; z, t)\right. \\
& \left.+\partial_{z}^{c} \mathcal{W}_{b a c}^{(3)}(y, v ; x, u ; z, t)\right) \\
& -2 i\left[\delta(z-x) \delta(t-u) * \mathcal{W}_{a b}^{(2)}(z, t ; y, v)\right] \\
& -2 i\left[\delta(z-y) \delta(t-v) * \mathcal{W}_{b a}^{(2)}(z, t ; x, u)\right],
\end{aligned}
$$


and so on. In (25) $\mathcal{W}_{a b}^{(2)}(x, u ; y, v)=\mathcal{W}_{b a}^{(2)}(y, v ; x, u)$, due to the cyclic symmetry.

Equation (21) guarantees the gauge invariance of the effective action. The problem now is how to compute the amplitudes $\mathcal{W}^{(n)}$. Here we will not follow the method introduced in [15], but mostly the more familiar Feynman diagram method, therefore it is more convenient to pass to the Fourier transforms. To start with we recall that, due to translational invariance,

$$
\begin{aligned}
\mathcal{W}_{a_{1} \ldots a_{n}}^{(n)}\left(x_{1}, u_{1}, \ldots, x_{n}, u_{n}\right) \\
\quad=\mathcal{W}_{a_{1} \ldots, a_{n}}^{(n)}\left(x_{1}-x_{n}, u_{1}, \ldots, 0, u_{n}\right) \\
\quad \equiv \mathcal{W}_{a_{1} \ldots a_{n}}^{(n)}\left(x_{1}-x_{n} \ldots, x_{n-1}-x_{n} ; u_{1}, \ldots, u_{n}\right) \\
\quad=i^{n-1}\left\langle J_{a_{1}}\left(x_{1}, u_{1}\right) \ldots J_{a_{n}}\left(x_{n}, u_{n}\right)\right\rangle \\
\quad=i^{n-1}\left\langle J_{a_{1}}\left(x_{1}-x_{n}, u_{1}\right) \ldots J_{a_{n}}\left(0, u_{n}\right)\right\rangle .
\end{aligned}
$$

The Fourier transform of this amplitude is

$$
\begin{aligned}
\int d^{d} & x_{1} e^{i k_{1} \cdot x_{1}} \ldots \int d^{d} x_{n-1} e^{i k_{n-1} \cdot x_{n-1}} \\
& \times \int d^{d} x_{n} e^{-i q \cdot x_{n}} \mathcal{W}_{a_{1} \ldots a_{n}}^{(n)}\left(x_{1}, u_{1}, \ldots, x_{n}, u_{n}\right) \\
= & \int d^{d} x_{1} e^{i k_{1} \cdot x_{1}} \ldots \int d^{d} x_{n-1} e^{i k_{n-1} \cdot x_{n-1}} \int d^{d} x_{n} e^{-i q \cdot x_{n}} \\
& \times \mathcal{W}_{a_{1} \ldots a_{n}}^{(n)}\left(x_{1}-x_{n}, \ldots, x_{n-1}-x_{n} ; u_{1}, \ldots, u_{n}\right) \\
= & \delta\left(q-\sum_{i=1}^{n-1} k_{i}\right) \int d^{d} x_{1}^{\prime} e^{i k_{1} \cdot x_{1}^{\prime}} \ldots, \\
\times & \int d^{d} x_{n-1}^{\prime} e^{i k_{n-1} \cdot x_{n-1}^{\prime}} \mathcal{W}_{a_{1} \ldots a_{n}}^{(n)}\left(x_{1}^{\prime} \ldots, x_{n-1}^{\prime} ; u_{1}, \ldots, u_{n}\right) \\
= & \delta\left(q-\sum_{i=1}^{n-1} k_{i}\right) \widetilde{\mathcal{W}}_{a_{1} \ldots a_{n}}^{(n)}\left(k_{1} \ldots, k_{n-1} ; u_{1}, \ldots, u_{n}\right) \\
= & i^{n-1} \delta\left(q-\sum_{i=1}^{n-1} k_{i}\right)\left\langle\widetilde{J}_{a_{1}}\left(k_{1}, u_{1}\right)\right. \\
& \left.\ldots \widetilde{J}_{a_{n-1}}\left(k_{n-1}, u_{n-1}\right) \widetilde{J}_{a_{n}}\left(-q, u_{n}\right)\right\rangle .
\end{aligned}
$$

In particular, for later reference,

$$
\begin{aligned}
& \int d^{d} x e^{i k \cdot x} \int d^{d} y e^{-i q \cdot y} \mathcal{W}_{a b}^{(2)}(x, u ; y, v) \\
& =\delta(q-k) \widetilde{\mathcal{W}}_{a b}^{(2)}(k ; u, v) \\
& =i \delta(q-k)\left\langle\widetilde{J}_{a}(k, u) \widetilde{J}_{b}(-k, v)\right\rangle
\end{aligned}
$$

and

$$
\begin{aligned}
\int d^{d} x e^{i k_{1} \cdot x} \int d^{d} y e^{i k_{2} \cdot y} \int d^{d} z e^{-i q \cdot z} \mathcal{W}_{a b c}^{(3)}(x, u ; y, v ; z, t) \\
=\delta\left(q-k_{1}-k_{2}\right) \widetilde{\mathcal{W}}_{a b c}^{(3)}\left(k_{1}, k_{2} ; u, v, t\right) \\
=i^{2} \delta\left(q-k_{1}-k_{2}\right)\left\langle\widetilde{J}_{a}\left(k_{1}, u\right) \widetilde{J}_{b}\left(k_{2}, v\right) \widetilde{J}_{c}(-q, t)\right\rangle .
\end{aligned}
$$

The WI (24) gets transformed into

$k^{a} \widetilde{\mathcal{W}}_{a b}^{(2)}(k ; u, v)=i k^{a}\left\langle\widetilde{J}_{a}(k, u) \widetilde{J}_{b}(-k, v)\right\rangle=0$

while (25) becomes (dropping $i \delta\left(q-k_{1}-k_{2}\right)$ ):

$$
\begin{aligned}
0= & i q^{c}\left\langle\widetilde{J}_{a}\left(k_{1}, u\right) \widetilde{J}_{b}\left(k_{2}, v\right) \widetilde{J}_{c}(-q, t)\right\rangle \\
& +i q^{c}\left\langle\widetilde{J}_{b}\left(k_{2}, v\right) \widetilde{J}_{a}\left(k_{1}, u\right) \widetilde{J}_{c}(-q, t)\right\rangle \\
& -2\left[\delta\left(t-v+\frac{k_{1}}{2}\right)\left\langle\widetilde{J}_{a}\left(k_{1}, u\right) \widetilde{J}_{b}\left(-k_{1}, t-\frac{k_{2}}{2}\right)\right\rangle\right. \\
& -\delta\left(t-v-\frac{k_{1}}{2}\right)\left\langle\widetilde{J}_{a}\left(k_{1}, u\right) \widetilde{J}_{b}\left(-k_{1}, t+\frac{k_{2}}{2}\right)\right\rangle \\
& +\delta\left(t-u+\frac{k_{2}}{2}\right)\left\langle\widetilde{J}_{b}\left(k_{2}, v\right) \widetilde{J}_{a}\left(-k_{2}, t-\frac{k_{1}}{2}\right)\right\rangle \\
& \left.-\delta\left(t-u-\frac{k_{2}}{2}\right)\left\langle\widetilde{J}_{b}\left(k_{2}, v\right) \widetilde{J}_{a}\left(-k_{2}, t+\frac{k_{1}}{2}\right)\right\rangle\right] .
\end{aligned}
$$

Using (28) and (29) one can write this as

$$
\begin{aligned}
0= & q^{c}\left(\widetilde{\mathcal{W}}_{a b c}^{(3)}\left(k_{1}, k_{2} ; u, v, t\right)+\tilde{\mathcal{W}}_{b a c}^{(3)}\left(k_{2}, k_{1} ; v, u, t\right)\right) \\
& -2\left[\delta\left(t-v+\frac{k_{1}}{2}\right) \widetilde{\mathcal{W}}_{a b}^{(2)}\left(k_{1} ; u, t-\frac{k_{2}}{2}\right)\right. \\
& -\delta\left(t-v-\frac{k_{1}}{2}\right) \widetilde{\mathcal{W}}_{a b}^{(2)}\left(k_{1} ; u, t+\frac{k_{2}}{2}\right) \\
& +\delta\left(t-u+\frac{k_{2}}{2}\right) \tilde{\mathcal{W}}_{b a}^{(2)}\left(k_{2} ; v, t-\frac{k_{1}}{2}\right) \\
& \left.-\delta\left(t-u-\frac{k_{2}}{2}\right) \widetilde{\mathcal{W}}_{b a}^{(2)}\left(k_{2} ;, v, t+\frac{k_{1}}{2}\right)\right] .
\end{aligned}
$$

To extract the WI in components one must multiply by a suitable expression $\frac{\partial^{j}}{\partial u^{j}} \delta(u)$ and integrate over $u$. Similarly for $v$ and $t$.

For a derivation of (31) and (32), see Appendix A.

\section{The case $\mathcal{W}_{a}^{(1)} \neq 0$}

\subsection{A curved $L_{\infty}$ algebra}

It was shown in [15] that, underlying the effective action obtained by integrating out a fermion field coupled to external sources, there is an algebraic structure, which is unveiled once we consider the relevant equations of motion. The basic relations in this game are the eom's

$\mathcal{F}_{a}(x, u)=0$

where

$$
\begin{aligned}
\mathcal{F}_{a}(x, u) \equiv & \sum_{n=0}^{\infty} \frac{1}{n !} \int \prod_{i=1}^{n} d^{d} x_{i} \frac{d^{d} u_{i}}{(2 \pi)^{d}} \mathcal{W}_{a a_{1} \ldots a_{n}}^{(n+1)} \\
& \times\left(x, u, x_{1}, u_{1}, \ldots, x_{n}, u_{n}\right) \\
& \times h^{a_{1}}\left(x_{1}, u_{1}\right) \ldots h^{a_{n}}\left(x_{n}, u_{n}\right)
\end{aligned}
$$


and the covariance relation

$\delta_{\varepsilon} \mathcal{F}_{a}(x, u)=i\left[\varepsilon(x, u) \stackrel{*}{\mathcal{F}_{a}}(x, u)\right]$

which can also be rewritten as follows

$$
\begin{aligned}
i[\varepsilon & \left.\left.*\left\langle\mathcal{W}_{a}^{(n+1)}, h^{\otimes n}\right\rangle\right\rangle\right] \\
= & \frac{1}{n+1} \sum_{i=1}^{n+1}\left\langle\left\langle\mathcal{W}_{a a_{1} \ldots \mu_{i} \ldots a_{n+1}}^{(n+2)}, h^{a_{1}} \ldots \partial_{x}^{a_{i}} \varepsilon \ldots h^{a_{n+1}}\right\rangle\right\rangle \\
& -i \sum_{i=1}^{n}\left\langle\left\langle\mathcal{W}_{a a_{1} \ldots a_{i} \ldots \mu_{n}}^{(n+1)}, h^{a_{1}} \ldots\left[h^{a_{i} *}, \varepsilon\right] \ldots h^{a_{n}}\right\rangle\right\rangle .
\end{aligned}
$$

It was shown in section 3 of [15], that this allows us to define multilinear maps (products) $L_{j}, j=1, \ldots, \infty$ of degree $d_{j}=j-2$ among vector spaces $X_{i}$ of degree $i$, defined by the assignments $\varepsilon \in X_{0}, h_{a} \in X_{-1}$ and $\mathcal{F}_{a} \in$ $X_{-2}$, which satisfy the relations

$$
\begin{aligned}
& \sum_{i+j=n+1}(-1)^{i(j-1)} \sum_{\sigma}(-1)^{\sigma} \epsilon(\sigma ; x) L_{j} \\
& \quad \times\left(L_{i}\left(x_{\sigma(1)}, \ldots, x_{\sigma(i)}\right), x_{\sigma(i+1)}, \ldots, x_{\sigma(n)}\right)=0 .
\end{aligned}
$$

In this formula $\sigma$ denotes a permutation of the entries so that $\sigma(1)<\cdots \sigma(i)$ and $\sigma(i+1)<\cdots \sigma(n)$, and $\epsilon(\sigma ; x)$ is the Koszul sign. Nonvanishing examples of such products are

$$
\begin{aligned}
& L_{1}(\varepsilon)^{a}=\partial_{x}^{a} \varepsilon(x, u) \\
& L_{2}(\varepsilon, h)^{a}=-i\left[h^{a}(x, u)^{*} \varepsilon(x, u)\right]=-L_{2}(h, \varepsilon)^{a} \\
& L_{2}\left(\varepsilon_{1}, \varepsilon_{2}\right)=i\left[\varepsilon_{1}^{*}, \varepsilon_{2}\right]
\end{aligned}
$$

together with

$$
\begin{aligned}
L_{1}(h) & =\left\langle\left\langle\mathcal{W}_{a}^{(2)}, h\right\rangle\right\rangle \\
& =\int d^{d} x_{i} \frac{d^{d} u_{i}}{(2 \pi)^{d}} \mathcal{W}_{a a_{1}}^{(2)}\left(x, u, x_{1}, p_{1}\right) h^{a_{1}}\left(x_{1}, u_{1}\right)
\end{aligned}
$$

and $L_{n}\left(h_{1}, \ldots, h_{n}\right)$, see the definition in [15]. Assuming the one-point correlator $\mathcal{W}_{a}^{(1)}=0$, in [15] it was proved that the relation (36) are satisfied.

In many cases, however $\mathcal{W}_{a}^{(1)} \neq 0$. When this happens we have to introduce an additional 'product' $L_{0}$, besides the $L_{n}$ of $[15,26]$. The algebra in this case is called curved $L_{\infty} \cdot{ }^{6} L_{0}$ is defined simply by setting

$$
L_{0}=\mathcal{W}_{a}^{(1)} \text {. }
$$

Both sides of this equation have degree -2 , coherently with the fact that the degree of $L_{n}$ is $n-2$. In this case $L_{1}$ is not nilpotent and the defining property $L_{1}^{2}=0$ of the $L_{\infty}$ algebra is modified as follows

$L_{1}\left(L_{1}(v)\right)+L_{2}\left(L_{0}, v\right)=0$

6 The notion of curved $L_{\infty}$ algebra is due to Jim Stasheff (private communication). where $v \in X=X_{0} \oplus X_{-1} \oplus X_{-2}$. Due to the degree counting, this relation is only nontrivial when $v \in X_{0}$, i.e. when $v$ is $\varepsilon$. Now using Eq. (3.21) of [15], i.e.

$L_{2}(\varepsilon, E)=i[\varepsilon \stackrel{*}{*} E]$

where $E$ represents $\mathcal{F}_{a}$ or any of its homogeneous pieces, and recalling that $L_{1}(\varepsilon)^{a}(x, u)=\partial_{x}^{a} \varepsilon(x, u)$ and $L_{1}(h)_{a}=$ $\left\langle\left\langle\mathcal{W}_{a}^{(2)}, h\right\rangle\right\rangle$, this equation becomes

$i\left[\mathcal{W}_{a}^{(1)}, \varepsilon\right]+\left\langle\left\langle\mathcal{W}_{a b}^{(2)} h^{b}\right\rangle\right\rangle=0$.

This is precisely the case $n=0$ of (35). All the other $L_{\infty}$ defining relations remain unchanged, because, for instance, the relation

$L_{3} L_{0}-L_{2} L_{1}+L_{1} L_{2}=0$

is not a priori excluded by the degree counting, but in [15] we have proved that $L_{3}(E, *, *)=0$ is consistent for $E$ of degree -2 . And so on.

$L_{0}$ is called the curvature of the curved $L_{\infty}$ algebra.

In this case (30) in momentum space becomes

$$
\begin{aligned}
& k^{a} \tilde{\mathcal{W}}_{a b}^{(2)}(k ; u, v)+\delta(u-v) \\
& \quad \times\left(\mathcal{W}_{b}^{(1)}\left(0, u+\frac{k}{2}\right)-\mathcal{W}_{b}^{(1)}\left(0, u-\frac{k}{2}\right)\right)=0 .
\end{aligned}
$$

In this formula $\mathcal{W}_{b}^{(1)}(0, u) \equiv \mathcal{W}_{b}^{(1)}(x=0, u)$.

\subsection{WI for 2-pt functions}

The method introduced in [15] to calculate the current amplitudes is less manageable then the more familiar method based on Feynman diagrams. It is however more compact and may turn out to be useful in some instances. Here we would like to show in a simple example, the WI for 2-pt correlators, that it leads to the expected results.

For $n=1$ and assuming $\mathcal{W}_{a}^{(1)} \neq 0$, (24) becomes

$$
\begin{aligned}
0= & \frac{1}{(2 \pi)^{d}} \partial_{x}^{a} \mathcal{W}_{a b}^{(2)}\left(x, u ; y, u^{\prime}\right) \\
& -i\left[\delta(x-y) \delta\left(u-u^{\prime}\right) * \mathcal{W}_{b}^{(1)}(x, u)\right]
\end{aligned}
$$

where

$$
\begin{aligned}
\mathcal{W}_{a_{1}, \ldots, a_{n}}^{(n)}\left(x_{1}, u_{1}, \ldots, x_{n}, u_{n}\right) & \\
= & -N \frac{n !}{n} \int_{\epsilon}^{\infty} d t \int \prod_{i=1}^{n} d^{d} q_{i} \int_{-\infty}^{\infty} \frac{d \omega}{2 \pi} e^{i \omega t} \\
& \times \operatorname{tr}\left[\gamma_{a_{1}} \frac{1}{q_{1}+m^{\prime}} \gamma_{a_{2}} \cdots \gamma_{a_{n-1}} \frac{1}{q_{n-1}+m^{\prime}} \gamma_{a_{n}} \frac{1}{q_{n}+m^{\prime}}\right] \\
& \times \prod_{j=1}^{n} e^{i q_{j} \cdot\left(x_{j}-x_{j+1}\right)} \delta\left(u_{1}-\frac{q_{1}+q_{n}}{2}\right) \\
& \ldots \delta\left(u_{n}-\frac{q_{n-1}+q_{n}}{2}\right)
\end{aligned}
$$


and we introduced $m^{\prime}=m-i(\omega-i \varepsilon)$. In particular, for $n=1$ (48) becomes

$$
\begin{aligned}
\mathcal{W}_{b}^{(1)}(x, u)=-N & \int_{\epsilon}^{\infty} d t \int d^{d} q \int_{-\infty}^{\infty} \frac{d \omega}{2 \pi} e^{i \omega t} \\
& \times \operatorname{tr}\left[\gamma_{b} \frac{1}{q+m^{\prime}}\right] \delta(u-q) \\
= & -2^{\left\lfloor\frac{d}{2}\right\rfloor} i N \int_{-\infty}^{\infty} \frac{d \omega}{2 \pi} \frac{e^{i \omega \epsilon}}{\omega} \frac{u_{b}}{p^{2}-m^{\prime 2}} .
\end{aligned}
$$

Note that the above equation does not depend on $x$. We can write $\mathcal{W}_{v}^{(1)}(x, u)=\mathcal{W}_{v}^{(1)}(u)$. For $n=2$

$$
\begin{aligned}
\mathcal{W}_{a b}^{(2)}\left(x, u, y, u^{\prime}\right) \\
=-N \int_{\epsilon}^{\infty} d t \int d^{d} q_{1} d^{d} q_{2} \\
\quad \times \int_{-\infty}^{\infty} \frac{d \omega}{2 \pi} e^{i \omega t} \operatorname{tr}\left[\gamma_{a} \frac{1}{q_{1}+m^{\prime}} \gamma_{b} \frac{1}{q_{2}+m^{\prime}}\right] \\
\quad \times e^{i\left(q_{1}-q_{2}\right) \cdot(x-y)} \delta\left(u-\frac{q_{1}+q_{2}}{2}\right) \delta\left(u^{\prime}-\frac{q_{1}+q_{2}}{2}\right) .
\end{aligned}
$$

We can introduce new variables $q_{1}-q_{2}=k$ and $q_{1}=q$. Then, (52) reads

$$
\begin{aligned}
\mathcal{W}_{a b}^{(2)}\left(x, u, y, u^{\prime}\right) \\
=-N \int_{\epsilon}^{\infty} d t \int d^{d} q d^{d} k \int_{-\infty}^{\infty} \frac{d \omega}{2 \pi} e^{i \omega t} \\
\quad \times \operatorname{tr}\left[\gamma_{a} \frac{1}{q+m^{\prime}} \gamma_{b} \frac{1}{q-\not k+m^{\prime}}\right] \\
\quad \times e^{i k \cdot(x-y)} \delta\left(u-\frac{2 q-k}{2}\right) \delta\left(u^{\prime}-\frac{2 q-k}{2}\right) .
\end{aligned}
$$

After the evaluation of the trace and the integration over $t$ and $q$ we obtain

$$
\begin{aligned}
\mathcal{W}_{a b}^{(2)} & \left(x, u, y, u^{\prime}\right) \\
= & -2^{\left\lfloor\frac{d}{2}\right\rfloor} i N \int d^{d} k \int_{-\infty}^{\infty} \frac{d \omega}{2 \pi} \frac{e^{i \omega \epsilon}}{\omega} \\
& \times \frac{1}{\left(\left(u+\frac{k}{2}\right)^{2}-m^{\prime 2}\right)\left(\left(u-\frac{k}{2}\right)^{2}-m^{\prime 2}\right)} \\
& \times\left[2 u_{a} u_{b}-\frac{k_{a} k_{b}}{2}-\left(u^{2}-\frac{k^{2}}{4}-m^{\prime 2}\right) \eta_{a b}\right] \\
& \times e^{i k \cdot(x-y)} \delta\left(u-u^{\prime}\right) .
\end{aligned}
$$

Let us now focus on the first term in (47)

$$
\begin{aligned}
& \frac{1}{(2 \pi)^{d}} \partial_{x}^{a} \mathcal{W}_{a b}^{(2)}\left(x, u ; y, u^{\prime}\right) \\
& =-2^{\left\lfloor\frac{d}{2}\right\rfloor} i N \int \frac{d^{d} k}{(2 \pi)^{d}} \int_{-\infty}^{\infty} \frac{d \omega}{2 \pi} \frac{e^{i \omega \epsilon}}{\omega} \\
& \quad \times \frac{1}{\left(\left(u+\frac{k}{2}\right)^{2}-m^{\prime 2}\right)\left(\left(u-\frac{k}{2}\right)^{2}-m^{\prime 2}\right)}
\end{aligned}
$$

$$
\begin{aligned}
& \times\left[2 u_{a} u_{b}-\frac{k_{a} k_{b}}{2}-\left(u^{2}-\frac{k^{2}}{4}-m^{\prime 2}\right) \eta_{a b}\right] \\
& \times \partial_{x}^{a} e^{i k \cdot(x-y)} \delta\left(u-u^{\prime}\right) \\
= & 2^{\left\lfloor\frac{d}{2}\right\rfloor} N \int \frac{d^{d} k}{(2 \pi)^{d}} \int_{-\infty}^{\infty} \frac{d \omega}{2 \pi} \frac{e^{i \omega \epsilon}}{\omega} e^{i k \cdot(x-y)} \delta\left(u-u^{\prime}\right) \\
& \times \frac{\left[2(u \cdot k) p_{b}+\left(m^{\prime 2}-u^{2}-\frac{k^{2}}{4}\right) k_{b}\right]}{\left(\left(u+\frac{k}{2}\right)^{2}-m^{\prime 2}\right)\left(\left(u-\frac{k}{2}\right)^{2}-m^{\prime 2}\right)} .
\end{aligned}
$$

On the other hand, the second term in (47) is

$$
\begin{aligned}
&-i\left[\delta(x-y) \delta\left(u-u^{\prime}\right) * \mathcal{W}_{b}^{(1)}(x, u)\right] \\
&= \delta(x-y) \delta\left(u-u^{\prime}\right) \sum_{n=0}^{\infty} \frac{1}{(2 n+1) !} \\
& \times\left(\frac{i}{2}\right)^{2 n}\left(\overleftarrow{\partial} x \cdot \vec{\partial}_{u}\right)^{2 n+1} \mathcal{W}_{b}^{(1)}(u) \\
&= 2^{\left\lfloor\frac{d}{2}\right\rfloor} N \int \frac{d^{d} k}{(2 \pi)^{d}} \int_{-\infty}^{\infty} \frac{d \omega}{2 \pi} \frac{e^{i \omega \epsilon}}{\omega} e^{i k \cdot(x-y)} \delta\left(u-u^{\prime}\right) \\
& \times \sum_{n=0}^{\infty} \frac{1}{2^{2 n}(2 n+1) !}\left(k \cdot \vec{\partial}_{u}\right)^{2 n+1}\left(\frac{u_{b}}{u^{2}-m^{\prime 2}}\right) .
\end{aligned}
$$

Taking the derivatives w.r.t. $u$ gives

$$
\begin{aligned}
-i\left[\delta(x-y) \delta\left(u-u^{\prime}\right) * \mathcal{W}_{b}^{(1)}(x, u)\right] \\
=2^{\left\lfloor\frac{d}{2}\right\rfloor} N \int \frac{d^{d} k}{(2 \pi)^{d}} \int_{-\infty}^{\infty} \frac{d \omega}{2 \pi} \frac{e^{i \omega \epsilon}}{\omega} e^{i k \cdot(x-y)} \delta\left(u-u^{\prime}\right) \\
\quad \times \sum_{n=0}^{\infty} \sum_{j=0}^{n}\left(-4^{j-n}(k \cdot u)^{2 j} k^{2(n-j)}\left(m^{\prime 2}-u^{2}\right)^{-2-n-j}\right) \\
\quad \times\left(\left(\begin{array}{c}
n+j \\
2 j
\end{array}\right)\left(m^{\prime 2}-u^{2}\right) k_{b}+2\left(\begin{array}{c}
n+j+1 \\
2 j+1
\end{array}\right)(k \cdot u) u_{b}\right) .
\end{aligned}
$$

This can be resummed into

$$
\begin{aligned}
&-i\left[\delta(x-y) \delta\left(u-u^{\prime}\right) * \mathcal{W}_{b}^{(1)}(x, u)\right] \\
&=-2^{\left\lfloor\frac{d}{2}\right\rfloor} N \int \frac{d^{d} k}{(2 \pi)^{d}} \int_{-\infty}^{\infty} \frac{d \omega}{2 \pi} \frac{e^{i \omega \epsilon}}{\omega} e^{i k \cdot(x-y)} \delta\left(u-u^{\prime}\right) \\
& \times \frac{\left[2(u \cdot k) p_{b}+\left(m^{\prime 2}-u^{2}-\frac{k^{2}}{4}\right) k_{b}\right]}{\left(\left(u+\frac{k}{2}\right)^{2}-m^{\prime 2}\right)\left(\left(u-\frac{k}{2}\right)^{2}-m^{\prime 2}\right)} .
\end{aligned}
$$

Now, this term has exactly the opposite sign w.r.t. (53) and we see that (47) is indeed valid for 2-pt functions.

\section{Explicit calculations}

After studying the general properties of the current amplitudes in the previous sections, the next step involves the 
methods to explicitly compute such amplitudes. In fact in [15] rather explicit formulas were presented. Using such formulas in Appendix B we have calculated the 0- and 1-pt current correlators. However, to compute more complicated correlators we find it more practical to resort to the usual Feynman rules.

\subsection{Feynman rules}

The free part of the action (13) gives rise to the usual fermion propagator

$\frac{i}{\not p+m}$.

The interaction part $S_{i n t}$ is given by (14), $J_{a}(x, u)$ is given by (16) and $J_{a \mu_{1} \ldots \mu_{s-1}}^{(s)}(x)$ is defined by (17). Therefore

$$
\begin{aligned}
S_{i n t}= & \int d^{d} x \sum_{n, m=0}^{\infty} \frac{i^{n}(-i)^{m}}{2^{n+m} n ! m !} \partial^{n} \bar{\psi}(x) \gamma_{a} \partial^{m} \psi(x) \\
& \times h^{a \mu_{1} \ldots \mu_{n} \mu_{n+1} \ldots \mu_{n+m}}(x) \\
= & \int d^{d} x \sum_{s=1}^{\infty} \sum_{n=0}^{s-1} \frac{(-1)^{n}(-i)^{s-1}}{2^{s-1} n !(s-n-1) !} \partial_{\mu_{1}} \\
& \ldots \partial_{\mu_{n}} \bar{\psi}(x) \gamma_{a} \partial_{\mu_{n+1}} \ldots \partial_{\mu_{s-1}} \psi(x) h^{a \mu_{1} \ldots \mu_{s-1}}(x) .
\end{aligned}
$$

By replacing the fields with plane waves while keeping the tensor structure we can determine the $V_{f f h}$ vertex:

$$
V_{f f h}: \frac{i}{2^{s-1}(s-1) !}, \gamma_{\mu} \sum_{n=0}^{s-1} k_{\left(\mu_{1}\right.}^{\prime} \ldots k_{\mu_{n}}^{\prime} k_{\mu_{n+1}} \ldots k_{\left.\mu_{s-1}\right)}
$$

where $k$ is an incoming fermion momentum, while $k^{\prime}$ is an outgoing one. The momentum conservation $\left(\delta\left(q+k-k^{\prime}\right), q\right.$ incoming) at the vertex is understood. The vertex (59) can be easily derived from (14) and the first line of (16), by replacing $\bar{\psi}\left(x+\frac{z}{2}\right)$ with $e^{-i k^{\prime} \cdot\left(x+\frac{z}{2}\right)}, \psi\left(x-\frac{z}{2}\right)$ with $e^{i k \cdot\left(x-\frac{z}{2}\right)}$ and $h^{a}(x, u)$ with $h^{a}(u) e^{i q \cdot x}$. Performing the integrals one gets

$$
\begin{aligned}
i \delta\left(q+k-k^{\prime}\right) \int d^{d} z \frac{d^{d} p}{(2 \pi)^{d}} e^{i\left(p-\frac{k+k^{\prime}}{2}\right) \cdot z} \gamma_{a} h^{a}(p) \\
\quad=i \delta\left(q+k-k^{\prime}\right) \gamma_{a} h^{a}\left(\frac{k+k^{\prime}}{2}\right) \\
\quad=i \delta\left(q+k-k^{\prime}\right) \gamma_{a} \sum_{n=0}^{\infty} \frac{1}{2^{n} n !} h^{a \mu_{1} \ldots \mu_{n}}\left(k+k^{\prime}\right)_{\mu_{1}} \ldots\left(k+k^{\prime}\right)_{\mu_{n}}
\end{aligned}
$$

where $h^{a \mu_{1} \ldots \mu_{n}}$ is a constant tensor completely symmetric in $\mu_{1}, \ldots, \mu_{n}$. So one can write the vertex also as

$$
\begin{aligned}
& V_{f f h}: \quad V_{a \mu_{1} \ldots \mu_{s-1}}^{(s)}\left(k+k^{\prime}\right) \\
& =\frac{i}{2^{s-1}(s-1) !} \gamma_{a}\left(k+k^{\prime}\right)_{\left(\mu_{1} \ldots\left(k+k^{\prime}\right) \mu_{s-1}\right)}
\end{aligned}
$$

or, introducing two polarization vectors $m^{a}, n^{v}$,

$$
\begin{aligned}
& V_{f f h}: \quad V^{(s)}\left(m, n ; k+k^{\prime}\right) \\
& =\frac{i}{2^{s-1}((s-1) !)^{2}} \not h\left(n \cdot\left(k+k^{\prime}\right)\right)^{s-1} .
\end{aligned}
$$

We can also introduce the global vertex

$$
\begin{aligned}
\mathcal{V}\left(m, n ; k+k^{\prime}\right) & =\sum_{s=1}^{\infty} V^{(s)}\left(m, n ; k+k^{\prime}\right) \\
& =i \sum_{s=1}^{\infty} \frac{1}{2^{s-1}((s-1) !)^{2}} \not h\left(n \cdot\left(k+k^{\prime}\right)\right)^{s-1} \\
& =i \not h I_{0}\left(2 n \cdot\left(k+k^{\prime}\right)\right)
\end{aligned}
$$

where $I_{0}(z)$ is the modified Bessel function of second kind.

Next, in order to derive the Feynman rules, we couple the fermions to external currents $j, \bar{j}$ via an action term $\int(\bar{j} \psi+\bar{\psi} j)$ and define

$$
\begin{aligned}
Z[h ; \bar{j}, j] & =\int \mathcal{D} \bar{\psi} \mathcal{D} \psi e^{i\left(S_{0}+\int(\bar{j} \psi+\bar{\psi} j)\right)} e^{i S_{i n t}} \\
& =e^{i S_{i n t}\left(\bar{\psi}=i \frac{\delta}{\delta j}, \psi=-i \frac{\delta}{\delta \bar{j}}\right)} e^{\int \bar{j} P j}
\end{aligned}
$$

where $P$ is the propagator. Then we define the generator of connected Green functions

$W[h ; j, \bar{j}]=-i \log Z[h ; \bar{j}, j], \quad W[h]=\left.W[h ; j, \bar{j}]\right|_{j=\bar{j}=0}$.

Now, the connected Green functions of currents are given by

$$
\begin{aligned}
& i^{n-1}\left\langle J_{\mu_{1}^{(1)} \ldots \mu_{s_{1}}^{(1)}}^{\left(s_{1}\right)}\left(x_{1}\right) \ldots J_{\mu_{1}^{(n)} \ldots \mu_{s_{n}}^{(n)}}^{\left(s_{n}\right)}\left(x_{n}\right)\right\rangle \\
& =\left.\frac{\delta}{\delta h^{\mu_{1}^{(1)} \ldots \mu_{s_{1}}^{(1)}\left(x_{1}\right)}} \cdots \frac{\delta}{\delta h^{\mu_{1}^{(n)} \ldots \mu_{s_{n}}^{(n)}\left(x_{n}\right)}} W[h]\right|_{h=0} \\
& =\frac{i^{n-1}}{n ! n !} \frac{\delta}{\delta h^{\mu_{1}^{(1)} \ldots \mu_{s_{1}}^{(1)}\left(x_{1}\right)}} \cdots \frac{\delta}{\delta h^{\mu_{1}^{(n)} \ldots \mu_{s_{n}}^{(n)}\left(x_{n}\right)}} \\
& \times\left.\left(S_{\text {int }}\left(i \frac{\delta}{\delta j},-i \frac{\delta}{\delta \bar{j}} ; h\right)^{n}\left(\int \bar{j} P j\right)^{n}\right)_{c}\right|_{h, j, \bar{j}=0}
\end{aligned}
$$

where the subscript $c$ means that only the connected graphs are retained.

The first contribution is the tadpole or one-point function

$\left\langle n^{s-1} \cdot m \cdot J^{(s)}(0)\right\rangle=-\int \frac{d^{d} p}{(2 \pi)^{d}} \operatorname{tr}\left(\frac{1}{\not p+m} V^{(s)}(m, n ; 2 p)\right)$

where $n^{s} \cdot m \cdot \widetilde{J}^{(s)}(k)$ is the Fourier transform of $n^{\nu_{1}} \ldots n^{\nu_{s}} m^{a}$ $J_{a v_{1} \ldots v_{s-1}}^{(s)}(x)$. 
The two-point function is

$$
\begin{aligned}
\left\langle n_{1}^{s_{1}} \cdot m_{1} \cdot \widetilde{J}^{\left(s_{1}\right)}(k) n^{s} \cdot m \cdot \widetilde{J}^{(s)}(-k)\right\rangle \\
=-\int \frac{d^{d} p}{(2 \pi)^{d}} \operatorname{tr}\left(\frac{1}{\not p+m} V^{(s)}\left(m_{1}, n_{1} ; 2 p-k\right)\right. \\
\left.\quad \times \frac{1}{\not p-\not k+m} V^{\left(s_{1}\right)}(m, n ; 2 p-k)\right) .
\end{aligned}
$$

The three-point function is $\left(q=k_{1}+k_{2}\right)$

$$
\begin{aligned}
\left\langle n_{1}^{s_{1}} \cdot m_{1} \cdot \widetilde{J}^{\left(s_{1}\right)}\left(k_{1}\right) n_{2}^{s_{2}} \cdot m_{2} \cdot \widetilde{J}^{\left(s_{2}\right)}\left(k_{2}\right) n^{s} \cdot m \cdot \widetilde{J}^{(s)}(q)\right\rangle \\
=-\int \frac{d^{d} p}{(2 \pi)^{d}} \operatorname{tr}\left(\frac{1}{\not p+m} V^{\left(s_{1}\right)}\left(n_{1} ; 2 p-k_{1}\right)\right. \\
\quad \times \frac{1}{\not p-\not k_{1}+m} V^{\left(s_{2}\right)}\left(n_{2} ; 2 p-2 k_{1}-k_{2}\right) \\
\left.\quad \times \frac{1}{\not p-q+m} V^{(s)}(n ; 2 p-q)\right) .
\end{aligned}
$$

The divergences of these amplitudes can be evaluated by acting with $k \cdot \frac{\partial}{\partial n}$ in the case of (68) and with $q \cdot \frac{\partial}{\partial n}$ in the case of (69).

\subsection{A master field derivation of Feynman rules}

It is interesting to observe that one can get Feynman rules not only for the component currents, but for the master current $J_{\mu}(x, u)$ itself. One proceeds as follows. The free action can be written

$$
\begin{aligned}
S_{0}= & \int d^{d} x \bar{\psi}(x)(i \gamma \cdot \partial-m) \psi(x) \\
= & \int d^{d} x d^{d} z \frac{d^{d} u}{(2 \pi)^{d}} e^{i z \cdot u} \bar{\psi}\left(x+\frac{z}{2}\right) \\
& \times(i \gamma \cdot \partial-m) \psi\left(x-\frac{z}{2}\right)
\end{aligned}
$$

while the interaction part is

$$
\begin{aligned}
S_{i n t}= & \left\langle\left\langle J_{a}, h^{a}\right\rangle\right\rangle=\int d^{d} x \frac{d^{d} u}{(2 \pi)^{d}} J_{a}(x, u) h^{a}(x, u) \\
= & \int d^{d} x d^{d} z \frac{d^{d} u}{(2 \pi)^{d}} e^{i z \cdot u} \psi\left(x+\frac{z}{2}\right) \gamma_{a} \psi \\
& \times\left(x-\frac{z}{2}\right) h^{a}(x, u) .
\end{aligned}
$$

As before we define the $Z$ function

$Z[h ; \bar{j}, j]=\int \mathcal{D} \bar{\psi} \mathcal{D} \psi e^{i\left(S_{0}+\int(\bar{j} \psi+\bar{\psi} j)\right)} e^{i S_{i n t}}$,

where, however, now

$$
\begin{aligned}
& \int(\bar{j} \psi+\bar{\psi} j) \\
& =\int d^{d} x d^{d} z \frac{d^{d} u}{(2 \pi)^{d}} e^{i z \cdot u}\left(\bar{j}\left(x+\frac{z}{2}\right) \psi\left(x-\frac{z}{2}\right)\right.
\end{aligned}
$$

$$
\left.+\bar{\psi}\left(x+\frac{z}{2}\right) j\left(x-\frac{z}{2}\right)\right) .
$$

The factor $e^{i z \cdot u}$ enters the definition of the integration measure over $x, u$ and $z$. Next, as usual, one completes the square and integrates over $\psi$ and $\bar{\psi}$. As a result, apart from an overall constant, we get

$Z[h ; \bar{j}, j]=e^{i S_{\text {int }}\left(\bar{\psi}=i \frac{\delta}{\delta j}, \psi=-i \frac{\delta}{\delta \bar{j}}\right)} e^{\int \bar{j} P j}$

where

$$
\begin{aligned}
\int \bar{j} P j= & \int d^{d} x_{1} d^{d} x_{2} d^{d} z \frac{d^{d} u}{(2 \pi)^{d}} e^{i z \cdot u} \bar{j}\left(x_{1}-\frac{z}{2}\right) \\
& \times P\left(x_{1}-\frac{z}{2}, x_{2}+\frac{z}{2}\right) j\left(x_{2}+\frac{z}{2}\right)
\end{aligned}
$$

and $P$ is the propagator:

$$
P(x, y)=i \int \frac{d^{d} k}{(2 \pi)^{d}} \frac{e^{i k \cdot(x-y)}}{\not k+m} .
$$

The generator of connected Green functions is defined as above

$W[h ; j, \bar{j}]=-i \log Z[h ; \bar{j}, j], \quad W[h]=\left.W[h ; j, \bar{j}]\right|_{j=\bar{j}=0}$.

The correlators of the master current $J_{a}(x, u)$ are obtained by differentiating $W$ with respect to $h^{a}(x, u)$. The Feynman rules have the same combinatorics as in an ordinary field theory, but the propagators and vertices are different.

\subsubsection{1-Point function}

$$
\begin{aligned}
\left\langle J_{a}(x, u)\right\rangle= & \int d^{d} z e^{i z \cdot u}\left\langle\bar{\psi}\left(x+\frac{z}{2}\right) \gamma_{a} \psi\left(x-\frac{z}{2}\right)\right\rangle \\
= & \int d^{d} z e^{i z \cdot u} \frac{\delta}{\delta j\left(x+\frac{z}{2}\right)} \gamma_{a} \frac{\delta}{\delta \bar{j}\left(x-\frac{z}{2}\right)} \\
& \times \int d^{d} y_{1} d^{d} y_{2} \frac{d^{d} v}{(2 \pi)^{d}} d^{d} t e^{i v \cdot t} \bar{j}\left(y_{1}-\frac{t}{2}\right) \\
& \times P\left(y_{1}-\frac{t}{2}, y_{2}+\frac{t}{2}\right) j\left(y_{2}+\frac{t}{2}\right) \\
= & -\int d^{d} z e^{i z \cdot u} \int d^{d} y_{1} d^{d} y_{2} \frac{d^{d} v}{(2 \pi)^{d}} d^{d} t e^{i v \cdot t} \operatorname{tr} \\
& \times\left(\gamma_{a} P\left(y_{1}-\frac{t}{2}, y_{2}+\frac{t}{2}\right)\right) \\
& \times \delta\left(x-y_{1}-\frac{z-t}{2}\right) \delta\left(x-y_{2}+\frac{z-t}{2}\right) .
\end{aligned}
$$

Replacing (76) and simplifying

$$
\begin{aligned}
\left\langle J_{a}(x, u)\right\rangle & =-\int \frac{d^{d} k}{(2 \pi)^{d}} d^{d} z e^{i(u-k) \cdot z} \operatorname{tr}\left(\gamma_{a} \frac{i}{\not k+m}\right) \\
& =-\operatorname{tr}\left(\gamma_{a} \frac{i}{\not h+m}\right)=-2^{\left\lfloor\frac{d}{2}\right\rfloor} i \frac{u_{a}}{u^{2}-m^{2}} .
\end{aligned}
$$


Now, inverting (16), one gets the one-point correlators for component currents

$$
\begin{aligned}
\left\langle J_{a \mu_{1} \ldots \mu_{s-1}}^{(s)}(x)\right\rangle & =\frac{1}{(s-1) !} \int \frac{d^{d} u}{(2 \pi)^{d}}\left\langle J_{a}(x, u)\right\rangle u_{\mu_{1}} \ldots u_{\mu_{s-1}} \\
& =\frac{1}{(s-1) !} \int \frac{d^{d} u}{(2 \pi)^{d}} \frac{2^{\left\lfloor\frac{d}{2}\right\rfloor} u_{a}}{u^{2}-m^{2}} u_{\mu_{1}} \ldots u_{\mu_{s-1}} .
\end{aligned}
$$

The Fourier transform of this amplitude corresponds to (67).

\subsubsection{2-Point function}

The connected 2-pt function for master currents is

$$
\begin{aligned}
\left\langle J_{a}(x,\right. & \left.u) J_{b}(y, v)\right\rangle \\
= & \int d^{d} z e^{i z \cdot u} \frac{\delta}{\delta j\left(x+\frac{z}{2}\right)} \gamma_{a} \frac{\delta}{\delta \bar{j}\left(x-\frac{z}{2}\right)} \\
& \times \int d^{d} t e^{i t \cdot v} \frac{\delta}{\delta j\left(y+\frac{t}{2}\right)} \gamma_{b} \frac{\delta}{\delta \bar{j}\left(y-\frac{t}{2}\right)} \\
& \times \frac{1}{4} \int \frac{d^{d} u_{1}}{(2 \pi)^{d}} d^{d} z_{1} e^{i z_{1} \cdot u_{1}} \int d x_{1} d x_{2} \bar{j}\left(x_{1}-\frac{z_{1}}{2}\right) \\
& \times P_{1,2} j\left(x_{2}+\frac{z_{1}}{2}\right) \\
& \times \int \frac{d^{d} u_{2}}{(2 \pi)^{d}} d^{d} z_{2} e^{i z_{2} \cdot u_{2}} \int d x_{3} d x_{4} \bar{j}\left(x_{3}-\frac{z_{2}}{2}\right) \\
& \times P_{3,4} j\left(x_{4}+\frac{z_{2}}{2}\right)
\end{aligned}
$$

where

$$
\begin{aligned}
& P_{1,2}=i \int \frac{d^{d} k_{1}}{(2 \pi i)^{d}} \frac{e^{i k_{1} \cdot\left(x_{1}-x_{2}-z_{1}\right)}}{k_{1}+m}, \\
& P_{3,4}=i \int \frac{d^{d} k_{2}}{(2 \pi i)^{d}} \frac{e^{i k_{2} \cdot\left(x_{3}-x_{4}-z_{2}\right)}}{k_{2}+m} .
\end{aligned}
$$

After integrating the delta functions coming from the $j, \bar{j}$ differentiations, one gets

$$
\begin{aligned}
\left\langle J_{a}(x,\right. & \left.u) J_{b}(y, v)\right\rangle \\
= & -\int d^{d} z d^{d} t \frac{d^{d} u_{1}}{(2 \pi)^{d}} \frac{d^{d} u_{2}}{(2 \pi)^{d}} d^{d} z_{1} d^{d} z_{2} e^{i(z \cdot u+t \cdot v)} \\
& e^{i\left(z_{1} \cdot u_{1}+z_{2} \cdot u_{2}\right)} \\
& \times \int \frac{d^{d} k_{1}}{(2 \pi)^{d}} \frac{d^{d} k_{2}}{(2 \pi)^{d}} e^{\left(k_{1}-k_{2}\right) \cdot(x-y)} e^{-i\left(k_{1}+k_{2}\right) \frac{t+z}{2}} \\
& \times \operatorname{tr}\left(\gamma_{a} \frac{i}{\not k_{1}+m} \gamma_{b} \frac{i}{k_{2}+m}\right) \\
= & -\int d^{d} z d^{d} t \int \frac{d^{d} k_{1}}{(2 \pi)^{d}} \frac{d^{d} k_{2}}{(2 \pi)^{d}} e^{\left(k_{1}-k_{2}\right) \cdot(x-y)} \\
& \times e^{i z\left(u-\frac{k_{1}+k_{2}}{2}\right)} e^{i t\left(v-\frac{k_{1}+k_{2}}{2}\right)} \operatorname{tr}\left(\gamma_{a} \frac{i}{k_{1}+m} \gamma_{b} \frac{i}{k_{2}+m}\right)
\end{aligned}
$$

setting $k_{1}=p, k_{2}=p-k$. Integrating out the delta functions

$$
\begin{aligned}
\left\langle J_{a}(x, u) J_{b}(y, v)\right\rangle & \\
= & -\int \frac{d^{d} k}{(2 \pi)^{d}} e^{i k \cdot(x-y)} \int \frac{d^{d} p}{(2 \pi)^{d}} \\
& \times \delta\left(u-\frac{2 p-k}{2}\right) \delta\left(v-\frac{2 p-k}{2}\right) \\
& \times \operatorname{tr}\left(\gamma_{a} \frac{i}{\not p+m} \gamma_{b} \frac{i}{\not p-\not k+m}\right) .
\end{aligned}
$$

In components

$$
\begin{aligned}
&\left\langle J_{a \mu_{1} \ldots \mu_{s-1}}^{(s)}(x) J_{b v_{1} \ldots v_{r-1}}^{(r)}(y)\right\rangle \\
&=-\frac{1}{(s-1) !(r-1) !} \int \frac{d^{d} u}{(2 \pi)^{d}} \frac{d^{d} v}{(2 \pi)^{d}} \\
& \times\left\langle J_{a}(x, u) J_{b}(y, v)\right\rangle u_{\mu_{1}} \ldots u_{\mu_{s-1}} v_{\nu_{1}} \ldots v_{v_{r-1}} \\
&=-\frac{1}{2^{s+r-2}(s-1) !(r-1) !} \int \frac{d^{d} p}{(2 \pi)^{d}} \\
& \times \int \frac{d^{d} k}{(2 \pi)^{d}} e^{i k \cdot(x-y)}(2 p-k)_{\mu_{1}} \ldots(2 p-k)_{\mu_{s-1}} \\
& \times(2 p-k)_{\nu_{1}} \ldots(2 p-k)_{v_{r-1}} \\
& \times \operatorname{tr}\left(\gamma_{a} \frac{i}{\not p+m} \gamma_{b} \frac{i}{\not p-\not k+m}\right) .
\end{aligned}
$$

Fourier transforming the above amplitude gives (68).

\subsubsection{3-Point function}

The connected 3-pt function for master currents is

$$
\begin{aligned}
\left\langle J_{a_{1}}\left(x_{1}, u_{1}\right) J_{a_{2}}\left(x_{2}, u_{2}\right) J_{a_{3}}\left(x_{3}, u_{3}\right)\right\rangle \\
=\frac{1}{3 !} \int d^{d} z_{1} e^{i z_{1} \cdot u_{1}} \frac{\delta}{\delta j\left(x_{1}+\frac{z_{1}}{2}\right)} \gamma_{a_{1}} \frac{\delta \bar{j}\left(x_{1}-\frac{z_{1}}{2}\right)}{\delta} \\
\quad \times \int d^{d} z_{2} e^{i z_{2} \cdot u_{2}} \frac{\delta}{\delta j\left(x_{2}+\frac{z_{2}}{2}\right)} \gamma_{a_{2}} \frac{\delta \bar{j}\left(x_{2}-\frac{z_{2}}{2}\right)}{\delta} \\
\quad \times \int d^{d} z_{3} e^{i z_{3} \cdot u_{3}} \frac{\delta}{\delta j\left(x_{3}+\frac{z_{3}}{2}\right)} \gamma_{a_{3}} \frac{\delta \bar{j}\left(x_{3}-\frac{z_{3}}{2}\right)}{\delta y^{2}} \\
\quad \times \int d^{d} y_{1} d^{d} \bar{y}_{1} \int d^{d} y_{2} d^{d} \bar{y}_{2} \int d^{d} y_{3} d^{d} \bar{y}_{3} \\
\int \frac{d^{d} v_{1}}{(2 \pi)^{d}} \frac{d^{d} v_{2}}{(2 \pi)^{d}} \frac{d^{d} v_{3}}{(2 \pi)^{d}} d^{d} t_{1} d^{d} t_{2} d^{d} t_{3} e^{i t_{1} \cdot v_{1}} e^{i t_{2} \cdot v_{2}} e^{i t_{3} \cdot v_{3}} \\
\quad \times \bar{j}\left(y_{1}-\frac{t_{1}}{2}\right) P_{1, \overline{1}} j\left(\bar{y}_{1}+\frac{t_{1}}{2}\right) \bar{j}\left(y_{2}-\frac{t_{2}}{2}\right) \\
\quad \times P_{2, \overline{2}} j\left(\bar{y}_{2}+\frac{t_{2}}{2}\right) \bar{j}\left(y_{3}-\frac{t_{3}}{2}\right) P_{3, \overline{3}} j\left(\bar{y}_{3}+\frac{t_{3}}{2}\right)
\end{aligned}
$$

where

$$
P_{i, \bar{i}}=i \int \frac{d^{d} k_{i}}{(2 \pi)^{d}} \frac{e^{i k_{i} \cdot\left(y_{i}-\bar{y}_{i}-t_{i}\right)}}{k_{i}+m}, \quad i=1,2,3 .
$$


Proceeding as before one finds

$$
\begin{aligned}
& \left\langle J_{a_{1}}\left(x_{1}, u_{1}\right) J_{a_{2}}\left(x_{2}, u_{2}\right) J_{a_{3}}\left(x_{3}, u_{3}\right)\right\rangle \\
& =i \int d^{d} z_{1} d^{d} z_{2} d^{d} z_{3} e^{i\left(z_{1} \cdot u_{1}+z_{2} \cdot u_{2}+z_{3} \cdot u_{3}\right)} \\
& \quad \times \int \prod_{i=1}^{3} \frac{d^{d} k_{i}}{(2 \pi)^{d}} e^{i k_{1} \cdot\left(x_{1}-x_{2}-\frac{z_{1}+z_{2}}{2}\right)} \\
& \quad \times e^{i k_{2} \cdot\left(x_{2}-x_{3}-\frac{z_{2}+z_{3}}{2}\right)} e^{i k_{3} \cdot\left(x_{3}-x_{1}-\frac{z_{3}+z_{1}}{2}\right)} \\
& \quad \times \operatorname{tr}\left(\gamma_{a_{1}} \frac{1}{k_{1}+m} \gamma_{a_{2}} \frac{1}{k_{2}+m} \gamma_{a_{3}} \frac{1}{k_{3}+m}\right) .
\end{aligned}
$$

Rearranging the terms and defining

$k_{1}=p, \quad k_{1}-k_{2}=q_{1}, \quad k_{2}-k_{3}=q_{2}$

$q_{1}, q_{2}$ are the momenta of two external outgoing legs. The third has ingoing momentum $q_{1}+q_{2}$. Finally one gets

$$
\begin{aligned}
& \left\langle J_{a_{1}}\left(x_{1}, u_{1}\right) J_{a_{2}}\left(x_{2}, u_{2}\right) J_{a_{3}}\left(x_{3}, u_{3}\right)\right\rangle \\
& =i \int \frac{d^{d} q_{1}}{(2 \pi)^{d}} \frac{d^{d} q_{2}}{(2 \pi)^{d}} e^{i\left(q_{1}+q_{2}\right) \cdot x_{1}} e^{-i q_{1} \cdot x_{2}} e^{-i q_{2} \cdot x_{3}} \\
& \quad \times \delta\left(u_{1}-\frac{2 p-q_{1}-q_{2}}{2}\right) \delta\left(u_{2}-\frac{2 p-q_{1}}{2}\right) \\
& \quad \times \delta\left(u_{3}-\frac{2 p-2 q_{1}-q_{2}}{2}\right) \\
& \quad \times \int \frac{d^{d} p}{(2 \pi)^{d}} \operatorname{tr}\left(\gamma_{a_{1}} \frac{1}{\not p+m} \gamma_{a_{2}} \frac{1}{\not p-q_{1}+m} \gamma_{a_{3}}\right. \\
& \left.\quad \times \frac{1}{\not p-q_{1}-q_{2}+m}\right) .
\end{aligned}
$$

To this one must add the cross term. The Fourier transform of this amplitude corresponds to (69).

\subsection{4 n-Point function}

Guess for the n-point function for master currents:

$$
\begin{aligned}
& \left\langle J_{a_{1}}\left(x_{1}, u_{1}\right) \ldots J_{a_{n}}\left(x_{n}, u_{n}\right)\right\rangle \\
& =-i^{n} \int \frac{d^{d} p}{(2 \pi)^{d}} \int \prod_{i=1}^{n-1} \frac{d^{d} q_{i}}{(2 \pi)^{d}} e^{i\left(q_{1}+\cdots+q_{n-1}\right) \cdot x_{1}} \\
& \quad \times e^{-i q_{1} \cdot x_{2}} \ldots e^{-i q_{n-1} \cdot x_{n}} \\
& \quad \times \delta\left(u_{1}-\frac{2 p-q_{1}-\cdots-q_{n-1}}{2}\right) \delta\left(u_{2}-\frac{2 p-q_{1}}{2}\right) \\
& \quad \ldots \delta\left(u_{n} \frac{2 p-2 q_{1}-\cdots-q_{n-1}}{2}\right) \\
& \quad \times \operatorname{tr}\left(\gamma_{a_{1}} \frac{1}{\not p+m} \gamma_{a_{2}} \frac{1}{\not p-q_{1}+m}\right. \\
& \left.\quad \ldots \gamma_{a_{n}} \frac{1}{\not p-q_{1}-\cdots-q_{n-1}+m}\right)
\end{aligned}
$$

$q_{1}, \ldots, q_{n-1}$ are outgoing external leg momenta, the $n$-th leg has momentum $q_{1}+\cdots+q_{n-1}$. To (90) one must add the contributions with permutation of $q_{1}, \ldots, q_{n-1}$.

Before closing this section let us remark that the integrals (85) are of the type already encountered and explicitly computed in $[33,34]$. They can be easily analysed with the dimensional regularization. It is likely that also the three-point functions can be successfully dealt with analogous techniques [44-47].

\section{Part II. Relation between the scalar and fermion model} In the second part of the paper we compare objects and properties of the matter fermion model with those of the scalar model. The relation between the two amounts to a quadratic relation between the sources of the former and the latter.

\section{About HS gauge symmetries}

We compare first the symmetry transformations in the scalar and in the fermion model.

\subsection{The gauge transformation in the scalar model}

In the scalar model the action is $S_{(0)}+S_{\text {int }}$, where

$S_{0}=\int d^{d} x \partial_{\mu} \varphi^{*} \partial^{\mu} \varphi$,

and

$S_{\mathrm{int}}[J, h]=\sum_{s=0}^{\infty} \int d^{d} x \frac{1}{s !} J_{\mu_{1} \ldots \mu_{s}}^{(s)}(x) h^{(s) \mu_{1} \ldots \mu_{s}}(x)$

with the currents being chosen in the simple form

$$
\begin{aligned}
J_{\mu_{1} \ldots \mu_{s}}^{(s)}(x)= & (-)^{s^{i}} \frac{\frac{s}{s-2}_{2^{s}}}{\sum_{n=0}^{s}(-1)^{n}} \\
& \times\left(\begin{array}{c}
s \\
n
\end{array}\right) \partial_{\left(\mu_{1}\right.} \ldots \partial_{\mu_{n}} \varphi^{*} \partial_{\mu_{n+1}} \ldots \partial_{\left.\mu_{s}\right)} \varphi .
\end{aligned}
$$

The $h$ field is defined by

$$
h(x, u)=\sum_{s=0}^{\infty} h^{(s)}(x, u)=\sum_{s=0}^{\infty} \frac{1}{s !} h_{(s)}^{\mu_{1} \ldots \mu_{s}}(x) u_{\mu_{1}} \ldots u_{\mu_{s}}
$$

and its transformation is

$\delta_{\epsilon} h(x, u)=\left(u \cdot \partial_{x}\right) \epsilon(x, u)-\frac{i}{2}[h(x, u) \stackrel{*}{,} \epsilon(x, u)]$,

where the $*$-product is defined by

$\alpha(x, u) * \beta(x, u)=\alpha(x, u) e^{\frac{i}{2}\left(\overleftarrow{\partial}_{x} \cdot \vec{\partial}_{u}-\vec{\partial}_{x} \cdot \overleftarrow{\partial}_{u}\right)} \beta(x, u)$ 
More explicitly, representing

$$
\begin{aligned}
h(x, u)= & \Phi(x)+a^{\mu}(x) u_{\mu}+\frac{1}{2} h^{\mu \nu}(x) u_{\mu} u_{\nu} \\
& +\frac{1}{6} b^{\mu \nu \lambda}(x) u_{\mu} u_{\nu} u_{\lambda} \\
& +\frac{1}{4 !} d^{\mu \nu \lambda \rho}(x) u_{\mu} u_{\nu} u_{\lambda} u_{\rho}+\cdots
\end{aligned}
$$

and

$$
\begin{aligned}
\eta(x, u)= & \epsilon(x)+\xi^{\mu}(x) u_{\mu}+\frac{1}{2} \Lambda^{\mu \nu}(x) u_{\mu} u_{\nu} \\
& +\frac{1}{3 !} \Sigma^{\mu \nu \lambda}(x) u_{\mu} u_{\nu} u_{\lambda} \\
& +\frac{1}{4 !} P^{\mu \nu \lambda \rho}(x) u_{\mu} u_{\nu} u_{\lambda} u_{\rho}+\cdots
\end{aligned}
$$

we get the transformations

$\delta^{(0)} \Phi=0$

$\delta^{(0)} a^{\mu}=\partial^{\mu} \epsilon$

$\delta^{(0)} h^{\mu \nu}=\partial^{\mu} \xi^{v}+\partial^{v} \xi^{\mu}$

$\delta^{(0)} b^{\mu \nu \lambda}=\partial^{\mu} \Lambda^{\nu \lambda}+\partial^{\nu} \Lambda^{\mu \lambda}+\partial^{\lambda} \Lambda^{\mu \nu}$

where $\partial^{\mu}=\eta^{\mu \nu} \partial_{\nu}$, which comes from the first term in the RHS of (95), and

$$
\begin{aligned}
& \delta^{(1)} \Phi=\frac{1}{2} \xi \cdot \partial \Phi-\frac{1}{2} a \cdot \partial \epsilon+\frac{1}{48} b^{\nu_{1} \nu_{2} \nu_{3}} \partial_{\nu_{1}} \partial_{\nu_{2}} \partial_{\nu_{3}} \epsilon \\
& -\frac{1}{48} \Sigma^{v_{1} \nu_{2} \nu_{3}} \partial_{\nu_{1}} \partial_{\nu_{2}} \partial_{\nu_{3}} \Phi \\
& -\frac{1}{16} \partial_{\nu_{1}} h^{\nu_{2} \nu_{3}} \partial_{\nu_{2}} \partial_{\nu_{3}} \xi^{\nu_{1}}+\frac{1}{16} \partial_{\nu_{1}} \partial_{\nu_{2}} a^{\nu_{3}} \partial_{\nu_{3}} \Lambda^{\nu_{1} \nu_{2}} \\
& -\frac{1}{384} \partial_{\nu_{1}} \partial_{\nu_{2}} b^{\nu_{3} v_{4} \nu_{5}} \partial_{\nu_{3}} \partial_{\nu_{4}} \partial_{\nu_{5}} \Lambda^{\nu_{1} \nu_{2}} \\
& +\frac{1}{384} \partial_{\nu_{1}} \partial_{\nu_{2}} \Sigma^{\nu_{3} \nu_{4} \nu_{5}} \partial_{\nu_{3}} \partial_{\nu_{4}} \partial_{\nu_{5}} h^{\nu_{1} \nu_{2}} \\
& \delta^{(1)} a^{\mu}=\frac{1}{2} \xi \cdot \partial a^{\mu}+\frac{1}{2} \partial_{\rho} \Phi \Lambda^{\mu \rho}-\frac{1}{2} \partial_{\rho} \epsilon h^{\mu \rho}-\frac{1}{2} a \cdot \partial \xi^{\mu} \\
& +\frac{1}{48} b^{\nu_{1} \nu_{2} \nu_{3}} \partial_{\nu_{1}} \partial_{\nu_{2}} \partial_{\nu_{3}} \xi^{\mu}-\frac{1}{48} \Sigma^{\nu_{1} \nu_{2} \nu_{3}} \partial_{\nu_{1}} \partial_{\nu_{2}} \partial_{\nu_{3}} a^{\mu} \\
& -\frac{1}{16} \partial_{\nu_{1}} b^{\nu_{2} \nu_{3} \mu} \partial_{\nu_{2}} \partial_{\nu_{3}} \xi^{\nu_{1}}+\frac{1}{16} \partial_{\nu_{1}} \Sigma^{\nu_{2} \nu_{3} \mu} \partial_{\nu_{2}} \partial_{\nu_{3}} a^{\nu_{1}} \\
& -\frac{1}{16} \partial_{\nu_{1}} h^{\nu_{2} \nu_{3}} \partial_{\nu_{2}} \partial_{\nu_{3}} \Lambda^{\nu_{1} \mu}+\frac{1}{16} \partial_{\nu_{1}} \partial_{\nu_{2}} h^{\nu_{3} \mu} \partial_{\nu_{3}} \Lambda^{\nu_{1} \nu_{2}} \\
& -\frac{1}{384} \partial_{\nu_{1}} \partial_{\nu_{2}} \partial_{\nu_{3}} \Sigma^{\nu_{4} \nu_{5} \mu} \partial_{\nu_{4}} \partial_{\nu_{5}} b^{\nu_{1} \nu_{2} \nu_{3}} \\
& +\frac{1}{384} \partial_{\nu_{1}} \partial_{\nu_{2}} \partial_{\nu_{3}} b^{\nu_{4} \nu_{5} \mu} \partial_{\nu_{4}} \partial_{\nu_{5}} \Sigma^{\nu_{1} \nu_{2} \nu_{3}} \\
& \delta^{(1)} h^{\mu \nu}=\frac{1}{2} \xi \cdot \partial h^{\mu \nu}-\partial_{\rho} \xi^{(\mu} h^{\nu) \rho}+\partial_{\rho} a^{(\mu} \Lambda^{v) \rho} \\
& -\frac{1}{2} a \cdot \partial \Lambda^{\mu \nu}-\frac{1}{2} \partial_{\lambda} \epsilon b^{\lambda \mu \nu}+\frac{1}{2} \Sigma^{\mu \nu \rho} \partial_{\rho} \Phi \\
& +\frac{1}{48} b^{\nu_{1} \nu_{2} \nu_{3}} \partial_{\nu_{1}} \partial_{\nu_{2}} \partial_{\nu_{3}} \Lambda^{\mu \nu}-\frac{1}{96} \Sigma^{\nu_{1} \nu_{2} \nu_{3}} \partial_{\nu_{1}} \partial_{\nu_{2}} \partial_{\nu_{3}} h^{\mu \nu} \\
& -\frac{1}{8} \partial_{\nu_{1}} b^{\nu_{2} \nu_{3}(\mu} \partial_{\nu_{2}} \partial_{\nu_{3}} \Lambda^{\nu) \nu_{1}}+\frac{1}{8} \partial_{\nu_{1}} \Sigma^{\nu_{2} \nu_{3}(\mu} \partial_{\nu_{2}} \partial_{\nu_{3}} h^{\nu) \nu_{1}} \\
& +\frac{1}{16} \partial_{\nu_{1}} \partial_{\nu_{2}} b^{\nu_{3} \mu \nu} \partial_{\nu_{3}} \Lambda^{\nu_{1} \nu_{2}}-\frac{1}{16} \partial_{\nu_{1}} \partial_{\nu_{2}} \Sigma^{\nu_{3} \mu \nu} \partial_{\nu_{3}} h^{\nu_{1} \nu_{2}}
\end{aligned}
$$

$$
\begin{aligned}
\delta^{(1)} b^{\mu \nu \lambda}= & \frac{1}{2} \xi \cdot \partial b^{\mu \nu \lambda}-\frac{3}{2} \partial_{\rho} \xi^{(\mu} b^{\nu \lambda) \rho} \\
& +\frac{3}{2} \partial_{\rho} h^{(\mu \nu} \Lambda^{\lambda) \rho}-\frac{3}{2} h^{\rho(\mu} \partial_{\rho} \Lambda^{\nu \lambda)} \\
& -\frac{1}{2} a^{\rho} \partial_{\rho} \Sigma^{\mu \nu \lambda}+\frac{3}{2} \Sigma^{\rho(\mu \nu} \partial_{\rho} a^{\lambda)} \\
& -\frac{1}{96} \Sigma^{\nu_{1} \nu_{2} \nu_{3}} \partial_{\nu_{1}} \partial_{\nu_{2}} \partial_{\nu_{3}} b^{\mu \nu \lambda}+\frac{1}{96} b^{\nu_{1} \nu_{2} \nu_{3}} \partial_{\nu_{1}} \partial_{\nu_{2}} \partial_{\nu_{3}} \Sigma^{\mu \nu \lambda} \\
& -\frac{3}{16} \partial_{\nu_{1}} b^{\nu_{2} \nu_{3}(\mu} \partial_{\nu_{2}} \partial_{\nu_{3}} \Sigma^{\nu \lambda) \nu_{1}} \\
& +\frac{3}{16} \partial_{\nu_{1}} \Sigma^{\nu_{2} \nu_{3}(\mu} \partial_{\nu_{2}} \partial_{\nu_{3}} b^{\nu \lambda) \nu_{1}}
\end{aligned}
$$

which is the full contribution for the gauge transformations of spin 1,2 and 3, under the assumption that all fields of spin higher than 3 are disregarded.

These transformations suggest that the fields $\Phi, a^{\mu}, h^{\mu \nu}$ do not coincide with the standard scalar vector and metric fields $\tilde{\Phi}, \tilde{A}^{\mu}, \tilde{g}^{\mu \nu}$. In fact restricting to U(1) gauge and diff transformations, they transform as (99) and

$\delta^{(1)} \Phi=\frac{1}{2} \xi \cdot \partial \Phi-\frac{1}{2} a \cdot \partial \epsilon-\frac{1}{16} \partial_{\mu} h^{\nu \rho} \partial_{\nu} \partial_{\rho} \xi^{\mu}$

$\delta^{(1)} a^{\mu}=\frac{1}{2} \xi \cdot \partial a^{\mu}-\frac{1}{2} \partial_{\rho} \epsilon h^{\mu \rho}-\frac{1}{2} a \cdot \partial \xi^{\mu}$

$\delta^{(1)} h^{\mu \nu}=\frac{1}{2} \xi \cdot \partial h^{\mu \nu}-\frac{1}{2} \partial_{\rho} \xi^{\mu} h^{\rho v}-\frac{1}{2} \partial_{\rho} \xi^{v} h^{\rho \mu}$.

The standard fields instead transform as

$\delta^{(1)} \tilde{\Phi}=\zeta \cdot \partial \tilde{\Phi}$

$\delta^{(1)} \tilde{a}^{\mu}=\zeta \cdot \partial \tilde{a}^{\mu}-\partial_{\lambda} \zeta^{\mu} \tilde{a}^{\lambda}$

$\delta^{(1)} \tilde{g}^{\mu \nu}=\zeta \cdot \partial \tilde{g}^{\mu \nu}-\partial_{\lambda} \zeta^{\mu} \tilde{g}^{\lambda \nu}-\partial_{\lambda} \zeta^{\nu} \tilde{g}^{\mu \lambda}$.

We can reproduce the above transformations if we identify the fields as follows

$$
\begin{aligned}
& \Phi=-\frac{1}{4} \tilde{a}_{\mu} \tilde{a}^{\mu}-\frac{1}{32}\left(\partial_{\mu} \tilde{h}_{v \rho} \partial^{v} \tilde{h}^{\mu \rho}-\frac{1}{2} \partial_{\mu} \tilde{h}_{v \rho} \partial^{\mu} \tilde{h}^{v \rho}\right)+\ldots \\
& a^{\mu}=\tilde{g}^{\mu \nu} \tilde{a}_{v}=\tilde{a}^{\mu}-\frac{1}{2} \tilde{h}^{\mu \nu} \tilde{a}_{v} \\
& g^{\mu \nu} \equiv \eta^{\mu \nu}-\frac{1}{2} h^{\mu \nu}=\eta^{\mu \nu}-\frac{1}{2} \tilde{h}^{\mu \nu}
\end{aligned}
$$

together with $\xi^{\mu}=2 \zeta^{\mu}$. In (108) ellipses stand for terms of order 3 and higher in $\tilde{h}$. For the second term in the RHS reproduces the third term in the RHS of (102) up to terms quadratic in the field $\tilde{h}$. In fact a series in $\tilde{h}$ is required to reproduce (102) exactly. In Sect. 5.4 we shall show that a unique term can replace this series, but for this it will be necessary to introduce the vielbein.

Due to the above transformation properties it is natural to refer to the master field $h$ as a metric-like field and to the HS geometry resulting from integrating out scalar matter, as metric-like geometry. 
5.2 The HS gauge transformation in the fermion model

Let us come now to the HS gauge transformation from the fermion model. It comes from the invariance of the fermion action written in the operator form

$S=\langle\bar{\psi}|-\gamma \cdot(\widehat{P}-\widehat{H})-m| \psi\rangle$

where $\widehat{P}_{\mu}$ is the momentum operator whose symbol is the classical momentum $u_{\mu}$ and $\widehat{H}$ is an operator whose symbol is $h(x, u)$. For $(111)$ is trivially invariant under the operation

$S=\left\langle\bar{\psi}\left|\widehat{O} \widehat{O}^{-1} \widehat{G} \widehat{O} \widehat{O}^{-1}\right| \psi\right\rangle$

where $\widehat{G}=-\gamma \cdot(\widehat{P}-\widehat{H})-m$. So it is invariant under

$\widehat{G} \longrightarrow \widehat{O}^{-1} \widehat{G} \widehat{O}, \quad|\psi\rangle \longrightarrow \widehat{O}^{-1}|\psi\rangle$.

Writing $\widehat{O}=e^{-i \widehat{E}}$ we find the infinitesimal version.

$\delta|\psi\rangle=i \widehat{E}|\psi\rangle, \quad \delta\langle\bar{\psi}|=-i\langle\bar{\psi}| \widehat{E}$.

Passing from operators to symbols, the transformation of $h^{\mu}(x, u)$ is made of two pieces,

$\operatorname{Symb}([\gamma \cdot \widehat{P}, \widehat{E}])=\left[\gamma \cdot u^{*}, \varepsilon(x, u)\right]=-i \gamma \cdot \partial_{x} \varepsilon(x, u)$

and

$\operatorname{Symb}([\gamma \cdot \widehat{H}, \widehat{E}])=\left[\gamma \cdot h(x, u) *{ }^{*} \varepsilon(x, u)\right]$.

In these equations $\gamma \cdot \widehat{H}=\gamma^{a} \widehat{H}_{a}$, where $a$ will be understood as a flat index. Of course, since the background is flat, writing $\gamma^{a} H_{a}$ or $\gamma^{\mu} H_{\mu}$ is the same, but the first writing leads to the correct interpretation. So, from now on, we will use $h_{a}(x, u)$. In the sequel the index $a$ will play a special role. In terms of symbols, we thus rewrite (18) as

$$
\begin{aligned}
\delta_{\varepsilon} h_{a}(x, u) & =\partial_{a}^{x} \varepsilon(x, u)-i\left[h_{a}(x, u) \stackrel{*}{,} \varepsilon(x, u)\right] \\
& \equiv \mathcal{D}_{a}^{x *} \varepsilon(x, u) .
\end{aligned}
$$

Next, it is helpful to see all the above formulas in components. To avoid a proliferation of indices, let us write the expansion of $h_{a}(x, u)$ as

$$
\begin{aligned}
h_{a}(x, u)= & A_{a}(x)+\chi_{a}^{\mu}(x) u_{\mu}+\frac{1}{2} b_{a}^{\mu \nu} u_{\mu} u_{\nu} \\
& +\frac{1}{6} c_{a}^{\mu \nu \lambda} u_{\mu} u_{\nu} u_{\lambda}+\frac{1}{4 !} d_{a}^{\mu \nu \lambda \rho} u_{\mu} u_{\nu} u_{\lambda} u_{\rho} \\
& +\frac{1}{5 !} f_{a}^{\mu \nu \lambda \rho \sigma} u_{\mu} u_{\nu} u_{\lambda} u_{\rho} u_{\sigma}+\cdots
\end{aligned}
$$

Notice that in the expansion (15) the indices $\mu_{1}, \ldots, \mu_{n}$ are upper (contravariant), as it should be, because in the Weyl quantization procedure the momentum has lower index, since it must satisfy $\left[x^{\mu}, p_{v}\right]=i \delta_{v}^{\mu}$. Of course when the background metric is flat the indices $a$ and $\mu_{i}$ are on the same footing, but it is useful to keep them distinct.
Similarly we write

$$
\begin{aligned}
\varepsilon(x, u)= & \epsilon(x)+\xi^{\mu} u_{\mu}+\frac{1}{2} \Lambda^{\mu \nu} u_{\mu} u_{v}+\frac{1}{3 !} \Sigma^{\mu \nu \lambda} u_{\mu} u_{\nu} u_{\lambda} \\
& +\frac{1}{4 !} P^{\mu \nu \lambda \rho} u_{\mu} u_{\nu} u_{\lambda} u_{\rho}+\frac{1}{5 !} \Omega \cdot u^{5}+\cdots
\end{aligned}
$$

To avoid a proliferation of symbols we use for the component of $\varepsilon(x, u)$ the same symbols as for the expansion of $\eta(x, u)$ in the scalar model. As we shall see they are not the same (in fact $\eta=2 \varepsilon$ ). We invite the reader to remember the distinction.

The transformation (117) reads. to lowest order,

$\delta^{(0)} A_{a}=\partial_{a} \epsilon$

$\delta^{(0)} \chi_{a}^{v}=\partial_{a} \xi^{v}$

$\delta^{(0)} b_{a}{ }^{\nu \lambda}=\partial_{a} \Lambda^{\nu \lambda}$.

To first order we have

$$
\begin{aligned}
\delta^{(1)} A_{a}= & \xi \cdot \partial A_{a}-\partial_{\rho} \epsilon \chi_{a}^{\rho} \\
\delta^{(1)} \chi_{a}^{\nu}= & \xi \cdot \partial \chi_{a}^{v}-\partial_{\rho} \xi_{v} \chi_{a}^{\rho}+\partial^{\rho} A_{a} \Lambda_{\rho}{ }^{v}-\partial_{\lambda} \epsilon b_{a}{ }^{\lambda v} \\
\delta^{(1)} b_{a}^{\nu \lambda}= & \xi \cdot \partial b_{a}{ }^{\nu \lambda}-\partial_{\rho} \xi^{v} b_{a}{ }^{\rho \lambda}-\partial_{\rho} \xi^{\lambda} b_{a}{ }^{\rho \nu} \\
& +\partial_{\rho} \chi_{a}^{\nu} \Lambda^{\rho \lambda}+\partial_{\rho} \chi_{a}^{\lambda} \Lambda^{\rho v}-\chi_{a}^{\rho} \partial_{\rho} \Lambda_{\nu \lambda} .
\end{aligned}
$$

The next nontrivial order contains terms with three derivatives, and so on.

Let us denote now by $\tilde{A}_{a}$ the standard U(1) gauge field and by $\tilde{e}_{a}^{\mu}=\delta_{a}^{\mu}-\tilde{\chi}_{a}^{\mu}$ the standard inverse vielbein, and let us restrict to gauge and diff transformations alone, we have

$$
\begin{aligned}
\delta \tilde{A}_{a} & \equiv \delta\left(\tilde{e}_{a}^{\mu} \tilde{A}_{\mu}\right) \equiv \delta\left(\left(\delta_{a}^{\mu}-\tilde{\chi}_{a}^{\mu}\right) \tilde{A}_{\mu}\right) \\
& =\left(-\xi \cdot \partial \tilde{\chi}_{a}^{\mu}+\partial_{\lambda} \xi^{\mu} \tilde{\chi}_{a}^{\lambda}\right) \tilde{A}_{\mu}+\left(\delta_{a}^{\mu}-\tilde{\chi}_{a}^{\mu}\right)\left(\partial_{\mu} \epsilon+\xi \cdot \tilde{A}_{\mu}\right) \\
& \approx \partial_{a} \epsilon+\xi \cdot \tilde{A}_{a}-\tilde{\chi}_{a}^{\mu} \partial_{\mu} \epsilon
\end{aligned}
$$

and

$$
\begin{aligned}
\delta \tilde{e}_{a}^{\mu} \equiv \delta\left(\delta_{a}^{\mu}-\tilde{\chi}_{a}^{\mu}\right) & =\xi \cdot \partial \tilde{e}_{a}^{\mu}-\partial_{\lambda} \xi^{\mu} \tilde{e}_{a}^{\lambda} \\
& =-\xi \cdot \tilde{\chi}_{a}^{\mu}-\partial_{a} \xi^{\mu}+\partial_{\lambda} \xi^{\mu} \tilde{\chi}_{a}^{\lambda}
\end{aligned}
$$

so that

$\delta \tilde{\chi}_{a}^{\mu}=\xi \cdot \partial \tilde{\chi}_{a}^{\mu}+\partial_{a} \xi^{\mu}-\partial_{\lambda} \xi^{\mu} \tilde{\chi}_{a}^{\lambda}$

where we have retained only the terms at most linear in the fields. From the above we see that we can make the identifications

$A_{a}=\tilde{A}_{a}, \quad \chi_{a}^{\mu}=\tilde{\chi}_{a}^{\mu}$

The transformations (120), (121) are consistent with Riemannian geometry. Concerning the HS theory, it contains more than symmetric tensors: beside the completely symmetric $h^{a \mu_{1} \ldots \mu_{n}}$ it contains also a Lorentz representation in which the index $a$ and one of the other indices are antisymmetric.

This point deserves a further comment. The interpretation of $a$ in $h_{a}{ }^{\mu_{1} \ldots \mu_{n}}$ as a flat index almost calls for local 
Lorentz invariance in the effective action (in this regard see for instance [43]). This question is discussed in detail in II [42]. It is shown there that the action (13) does admit a local Lorentz invariant extension. As a consequence it is expected that the relevant Ward identities, barring anomalies, are obeyed, leading to Lorentz invariant effective actions. In II explicit examples are constructed. One of the consequences of this symmetry is that the antisymmetric part of $\chi_{a}^{\mu}$ can be identified with local Lorentz gauge parameters and thus eliminated by gauge fixing. As for the HS components of $h_{a}{ }^{\mu_{1} \ldots \mu_{n}}$ the problem is open. For instance in II it is shown that, in the YM-like models, in order to guarantee the existence of the relevant propagators, $h^{a \mu_{1} \ldots \mu_{n}}$ must be traceless in the $\mu_{i}$ indices. In general, however, this problem needs to be further investigated.

\subsection{Analogy with gauge transformations in gauge theories}

Notice first that, in Eqs. (117) and (120), the derivative $\partial_{a}$ means

$\partial_{a}=\delta_{a}^{\mu} \partial_{\mu}$,

not

$\partial_{a}=e_{a}^{\mu} \partial_{\mu}=\left(e_{a}^{\mu}-\chi_{a}^{\mu}+\cdots\right) \partial_{\mu}$,

for the linear correction $-\chi_{a}^{\mu} \partial_{\mu}$ is contained in the term $-i\left[h_{a}(x, u) \stackrel{*}{,} \varepsilon(x, u)\right]$, see for instance the second term in the RHS of the first equation (121). Therefore the transformation (117) looks similar to an ordinary gauge transformation of a non-Abelian gauge field

$\delta_{\lambda} A_{a}=\partial_{a} \lambda+\left[A_{a}, \lambda\right]$

where $A_{a}=A_{a}^{\alpha} T^{\alpha}, \lambda=\lambda^{\alpha} T^{\alpha}, T^{\alpha}$ being the Lie algebra generators.

In gauge theories it is useful to represent the gauge potential as a connection one form $\mathbf{A}=A_{a} d x^{a}$, so that (128) becomes

$\delta_{\lambda} \mathbf{A}=\mathbf{d} \lambda+[\mathbf{A}, \lambda]$.

We can do the same for (117)

$\delta_{\varepsilon} \mathbf{h}(x, u)=\mathbf{d} \varepsilon(x, u)-i[\mathbf{h}(x, u) \stackrel{*}{,} \varepsilon(x, u)] \equiv \mathbf{D}^{*} \varepsilon(x, u)$

where $\mathbf{d}=\partial_{a} d x^{a}, \mathbf{h}=h_{a} d x^{a}$ and $x^{a}$ are coordinates in the tangent spacetime, and it is understood that

$[\mathbf{h}(x, u) \stackrel{*}{,} \varepsilon(x, u)]=\left[h_{a}(x, u) \stackrel{*}{,} \varepsilon(x, u)\right] d x^{a}$.

Like in gauge theories it is straightforward to introduce the curvature

$G_{a b}=\partial_{a} h_{b}-\partial_{b} h_{a}-i\left[h_{a} \stackrel{*}{,} h_{b}\right]$ whose transformation rule is

$\delta_{\varepsilon} G_{a b}=-i\left[G_{a b} \stackrel{*}{,} \varepsilon\right]$,

as well as the curvature two-form

$\mathbf{G}=\mathbf{d h}-\frac{i}{2}[\mathbf{h} * \mathbf{h}]$,

with the transformation property

$\delta_{\varepsilon} \mathbf{G}=-i\left[\mathbf{G}^{*}, \varepsilon\right]$.

In part III of this paper this formalism will be applied to the construction of anomalies and in paper II to the construction of Yang-Mills and Chern-Simons-like theories.

The YM-like interpretation presented so far for $h_{a}$ and relevant constructs, using the analogy with gauge theories, is not the only possible one. There is another, which we have already dubbed geometry-like, which will be developed in the paper III.

\subsection{Connection between metric-like and frame-like master} fields

It is expected that there is a common set of higher spin fields, which couples both to the fermionic and bosonic matter, so it is important to find the connection between frame-like and metric like fields. One can show that if one defines the composite master field ${ }^{7}$

$h(x, u)=2 u_{a} h^{a}(x, u)-h_{a}(x, u) * h^{a}(x, u)$

and calculates its HS transformation by using (117), one gets

$\delta_{\varepsilon} h(x, u)=2\left(u \cdot \partial^{x}\right) \varepsilon(x, u)-i\left[h(x, u)^{*} \varepsilon(x, u)\right]$.

We see that it becomes identical to (95) if we make the identifications

$\eta(x, u)=2 \varepsilon(x, u)$.

The expression (135) defines the simplest metric-like master field constructed from the frame-like master field which satisfies the transformation law (95), and so can be used to "linearly" couple HS fields to bosonic matter. It is now obvious that coupling to bosonic matter is at least quadratic in powers of (fundamental) HS fields.

By using (135) we can now express metric-like spacetime fields in terms of frame-like spacetime fields by Taylor expanding both sides of the equation around $u=0$. For example, the scalar component is given by

$\Phi(x)=-\left.h_{a}(x, u) \star h^{a}(x, u)\right|_{u=0}$

\footnotetext{
7 In the forthcoming paper III we shall show that expression (135) naturally follows from the geometry-like formalism we develop there.
} 


$$
=-A_{a}(x) A^{a}(x)-\frac{1}{2} \partial_{\mu} \chi_{a}{ }^{\nu} \partial_{\nu} \chi^{a \mu}+\cdots
$$

where ellipses denote terms containing fields of spin $s \geq 3$. For spin 1 and 2 components one gets

$a^{\mu}(x)=2 e_{a}^{\mu}(x) A^{a}(x)+\cdots$

$h^{\mu \nu}(x)=2\left(\delta_{a}^{\mu} \chi^{a v}(x)+\delta_{a}^{v} \chi^{a \mu}(x)-\chi^{a \mu}(x) \chi_{a}{ }^{\nu}(x)\right)+\cdots$

where

$e_{a}^{\mu}(x) \equiv \delta_{a}^{\mu}-\chi_{a}^{\mu}(x)$.

The relations (139) and (140) are natural once we recognize $e_{a}^{\mu}(x)$ as vielbein and

$g^{\mu \nu}(x)=\eta^{\mu \nu}-\frac{1}{2} h^{\mu \nu}(x)$

as metric. The relation (140) then becomes

$g^{\mu \nu}(x)=\eta^{a b} e_{a}^{\mu}(x) e_{b}^{v}(x)$

which is the standard relation between (inverse) metric and vielbein.

As for (138), while the first term produces the standard "seagull" term in the Klein-Gordon coupling to the $U(1)$ gauge field, the second term is of more mysterious nature. Superficially it does not look locally Lorentz invariant, however, as we noticed in our comment after Eq. (110) it is likely to reproduce an infinite series in $h_{\mu \nu}$, and so to be local Lorentz invariant. Incidentally this tells us that the question of local Lorentz invariance in this formalism is rather subtle, see II. It also suggests that the coupling of Klein-Gordon field to HS fields apparently cannot be described in terms of Riemannian geometry. We shall say more on this in the paper III.

\section{Part III. Chern-Simons terms and anomalies}

The HS gauge transformation (18) suggests in an obvious way an analogy with gauge transformations in ordinary (nonAbelian) gauge theories. In both cases they are realized via Ward identities on the physical amplitudes. But as an effect of quantization the Ward identities in a second quantized theory may be violated, or anomalous. It is to be expected that a similar possibility exists also in the matter theories coupled to external HS sources introduced in this paper. In the third part we analyse the form of the possible obstructions that may appear in the Ward identities in such context.

\section{Obstructions}

In theory we can compute $\mathcal{W}^{(n)}$ by means of formula (2.52) of [15] or by means of Feynman diagrams. In practice we may find obstacles.

The first is the possibility that the one-point function does not vanish: $\mathcal{W}^{(1)} \neq 0$. In this case (24) is modified and the natural setting is a curved $L_{\infty}$.
The second is that the results one obtains may not satisfy the WI's. If it is so, however, we are helped by the consistency conditions. For, if

$\delta_{\varepsilon} \mathcal{W}[h]=\mathcal{A}[\varepsilon, h] \neq 0$,

as a consequence of

$$
\begin{aligned}
& \left(\delta_{\varepsilon_{2}} \delta_{\varepsilon_{1}}-\delta_{\varepsilon_{1}} \delta_{\varepsilon_{2}}\right) h^{\mu}(x, u) \\
& \left.\quad=i\left(\partial_{x}\left[\varepsilon_{1}, \varepsilon_{2}\right](x, u)-i\left[h^{\mu}(x, u) \stackrel{*}{,}\left[\varepsilon_{1}, * \varepsilon_{2}\right](x, u)\right]\right]\right) \\
& \quad=i \mathcal{D}_{x}^{* \mu}\left[\varepsilon_{1}, \varepsilon_{2}\right](x, u),
\end{aligned}
$$

we must have

$\delta_{\varepsilon_{2}} \mathcal{A}\left[\varepsilon_{1}, h\right]-\delta_{\varepsilon_{1}} \mathcal{A}\left[\varepsilon_{2}, h\right]=\mathcal{A}\left[\left[\varepsilon_{1}, \varepsilon_{2}\right], h\right]$.

The simplest possibility we may encounter is that $\mathcal{A}[\varepsilon, h]$ is trivial, i.e.

$\mathcal{A}[\varepsilon, h]=\delta_{\varepsilon} \mathcal{\varrho}[h]$,

where $\mathcal{C}[h]$ is an integrated local counterterm. In such a case we can recover invariance in the form

$\delta_{\varepsilon}(\mathcal{W}[h]-\mathrm{C}[h])=0$.

If however (147) is not true for any local choice of $\mathcal{C}[h]$, then we are faced with a true anomaly, which breaks the covariance of the effective action.

The case of trivial anomalies is what often occurs in Feynman diagram calculations when tadpoles or seagull diagrams are disregarded: the WI is violated, but invariance can be restored by suitable subtractions of local terms.

When, instead, (147) is not true for any local choice of $\mathcal{C}[h]$, it means that there is a true obstruction to building a gauge covariant theory. The true anomalies, i.e. the nontrivial cocycles (144), are the mathematical objects that classify such obstructions.

We can apply the frame-like formalism to the construction of CS actions and anomalies.

\subsection{HS CS terms}

The idea in this subsection is to mimic standard constructions of ordinary field theories in the framework of HS. For instance, beside (133) we can introduce the standard (ordinary gauge theories) definitions

$\mathbf{G}_{t}=\mathbf{d h}_{t}-\frac{i}{2}\left[\mathbf{h}_{t}, \mathbf{h}_{t}\right], \quad \mathbf{h}_{t}=t \mathbf{h}$.

Let us quote some formulas that will be useful later

$$
\begin{aligned}
\frac{d}{d t} \mathbf{G}_{t} & =\mathbf{d h}-i t[\mathbf{h} * \mathbf{h}]=\mathbf{d}_{t} \mathbf{h}, \quad \mathbf{d}_{t}=\mathbf{d}-i\left[\mathbf{h}_{t}, *\right] \\
\mathbf{d G}_{t} & =i\left[\mathbf{h}_{t}, \mathbf{G}_{t}\right], \quad \delta \mathbf{G}_{t}=\mathbf{d} \delta \mathbf{h}_{t}-i\left[\mathbf{h}_{t}, \delta \mathbf{h}_{t}\right]=\mathbf{d}_{t} \delta \mathbf{h}_{t} .
\end{aligned}
$$


The difference in the HS case is that, unlike in ordinary gauge theories, we cannot use graded commutativity. There is also another difficulty: in the HS case we don't have a trace at our disposal. The only object with trace properties we can define is

$$
\begin{aligned}
\langle\langle * g\rangle\rangle & =\int d^{d} x \int \frac{d^{d} u}{(2 \pi)^{d}} f(x, u) * g(x, u) \\
& =\int d^{d} x \int \frac{d^{d} u}{(2 \pi)^{d}} f(x, u) g(x, u)=\langle\langle g * f\rangle\rangle .
\end{aligned}
$$

From this, plus associativity, it follows that

$$
\begin{gathered}
\left\langle\left\langle f_{1} * f_{2} * \cdots * f_{n}\right\rangle\right\rangle=\left\langle\left\langle f_{1} *\left(f_{2} * \cdots * f_{n}\right)\right\rangle\right\rangle \\
=(-1)^{\epsilon_{1}\left(\epsilon_{2}+\cdots+\epsilon_{n}\right)}\left\langle\left\langle\left(f_{2} * \cdots * f_{n}\right) * f_{1}\right\rangle\right\rangle \\
=(-1)^{\epsilon_{1}\left(\epsilon_{2}+\cdots+\epsilon_{n}\right)}\left\langle\left\langle f_{2} * \cdots * f_{n} * f_{1}\right\rangle\right\rangle
\end{gathered}
$$

where $\epsilon_{i}$ is the Grassmann degree of $f_{i}$. In particular

$$
\left\langle\left\langle\left[f_{1} * f_{2} * \cdots * f_{n}\right\}\right\rangle\right\rangle=0
$$

where [ $\left.{ }^{*}\right]$ is the $*$-commutator or anti-commutator, as appropriate.

But in order to exploit this property we have to integrate over the full phase space. Therefore it is impossible to reproduce the unintegrated descent equations like in the ordinary gauge theories. The best we can do is to try to reproduce each equation separately in integrated form. So let us start from the phase space integral with $n \mathbf{G}$ entries

$\langle\langle\mathbf{G} * \mathbf{G} * \cdots * \mathbf{G}\rangle\rangle$.

Here $\langle\langle\rangle$ means integration over a phase space of dimension $4 n$. Then consider the expression with $n-1 \mathbf{G}_{t}$ entries

$$
\begin{aligned}
\int_{0}^{1} d t & \left\langle\left\langle\mathbf{d}\left(\mathbf{h} * \mathbf{G}_{t} * \cdots * \mathbf{G}_{t}\right)\right\rangle\right\rangle \\
= & \int_{0}^{1} d t\left\langle\left\langle\mathbf{d} \mathbf{h} * \mathbf{G}_{t} * \cdots * \mathbf{G}_{t}\right\rangle\right\rangle \\
& -\int_{0}^{1} d t\left\langle\left\langle\mathbf{h} * \mathbf{d} \mathbf{G}_{t} * \cdots * \mathbf{G}_{t}\right\rangle\right\rangle-\cdots \\
& -\int_{0}^{1} d t\left\langle\left\langle\mathbf{h} * \mathbf{G}_{t} * \cdots * \mathbf{d G}_{t}\right\rangle\right\rangle \\
= & \int_{0}^{1} d t\left(\left\langle\left\langle\mathbf{d} \mathbf{h} * \mathbf{G}_{t} * \cdots * \mathbf{G}_{t}\right\rangle\right\rangle\right. \\
& -i\left\langle\left\langle\mathbf{h} *\left[\mathbf{h}_{t} * \mathbf{G}_{t}\right] * \cdots * \mathbf{G}_{t}\right\rangle\right\rangle-\cdots \\
& \cdots-i\left\langle\left\langle\mathbf{h} * \mathbf{G}_{t} * \cdots *\left[\mathbf{h}_{t}, \mathbf{G}_{t}\right]\right)\right\rangle
\end{aligned}
$$

using the first of (151). Then using the first of (150) together with (153) (or (154)) one gets

$$
\begin{aligned}
\left.\int_{0}^{1} d t\left\langle\mathbf{d}\left(\mathbf{h} * \mathbf{G}_{t} * \cdots * \mathbf{G}_{t}\right)\right\rangle\right\rangle \\
\left.\quad=\int_{0}^{1} d t\left\langle\left(\mathbf{d h}-i\left[\mathbf{h}_{t} * \mathbf{h}\right]\right) * \mathbf{G}_{t} * \cdots * \mathbf{G}_{t}\right\rangle\right\rangle
\end{aligned}
$$

$$
\begin{aligned}
& =\int_{0}^{1} d t\left\langle\left\langle\left(\frac{d}{d t} \mathbf{G}_{t} * \mathbf{G}_{t} * \cdots * \mathbf{G}_{t}\right\rangle\right\rangle\right. \\
& =\frac{1}{n} \int_{0}^{1} d t \frac{d}{d t}\left\langle\left\langle\mathbf{G}_{t} * \mathbf{G}_{t} * \cdots * \mathbf{G}_{t}\right\rangle\right\rangle \\
& =\frac{1}{n}\langle\langle\mathbf{G} * \mathbf{G} * \cdots * \mathbf{G}\rangle\rangle .
\end{aligned}
$$

Since they are integrated over a spacetime of dimension $d=2 n$ these expressions vanish (unless the spacetime is topologically nontrivial), but this is the way we identify the primitive functional action for HS CS terms in dimension $d=2 n-1$ :

$\mathcal{C S}(\mathbf{h})=n \int_{0}^{1} d t\left\langle\left\langle\mathbf{h} * \mathbf{G}_{t} * \ldots * \mathbf{G}_{t}\right\rangle\right\rangle$

where $\langle\langle\rangle$ means now integration over a phase space of dimension $4 n-2$. The important thing is to prove that $C S(\mathbf{h})$ is invariant under the HS gauge transformation (130):

$$
\begin{aligned}
\delta_{\varepsilon} \mathcal{C S}(\mathbf{h})= & n \int_{0}^{1} d t\left(\left\langle\left\langle\mathbf{D} \varepsilon * \mathbf{G}_{t} * \cdots * \mathbf{G}_{t}\right\rangle\right\rangle\right. \\
& +t\left\langle\left\langle\mathbf{h} * \mathbf{d}_{t} \mathbf{D} \varepsilon * \cdots * \mathbf{G}_{t}\right\rangle\right\rangle+\cdots \\
& \left.\cdots+t\left\langle\left\langle\mathbf{h} * \mathbf{G}_{t} * \cdots * \mathbf{d}_{t} \mathbf{D} \varepsilon\right\rangle\right\rangle\right)
\end{aligned}
$$

Since $\mathbf{d}_{t} \mathbf{G}_{t}=0$ we can collect the symbol $\mathbf{d}_{t}$ and integrate by parts in space to obtain

$$
\begin{aligned}
\delta_{\varepsilon} \mathcal{C S}(\mathbf{h})= & n \int_{0}^{1} d t\left\{\left(\left\langle\mathbf{D} \varepsilon * \mathbf{G}_{t} * \cdots * \mathbf{G}_{t}\right\rangle\right\rangle\right. \\
& \left.+t \frac{d}{d t}\left\langle\left\langle\mathbf{D} \varepsilon * \mathbf{G}_{t} * \cdots * \mathbf{G}_{t}\right\rangle\right\rangle\right) \\
& -t\left\langle\left\langle\mathbf { d } \left(\mathbf{h} * \mathbf{D} \varepsilon * \mathbf{G}_{t} * \cdots * \mathbf{G}_{t}\right.\right.\right. \\
& +\mathbf{h} * \mathbf{G}_{t} * \mathbf{D} \varepsilon * \cdots * \mathbf{G}_{t}+\cdots \\
& \left.\left.\left.\left.\cdots+\mathbf{h} * \mathbf{G}_{t} * \cdots * \mathbf{G}_{t} * \mathbf{D} \varepsilon\right)\right\rangle\right\rangle\right\} .
\end{aligned}
$$

For a proof see Appendix D. Upon integrating by parts $\frac{d}{d t}$, the RHS of the first line becomes

$$
\begin{aligned}
n\langle\langle\mathbf{D} \varepsilon * \mathbf{G} * \cdots * \mathbf{G}\rangle\rangle & =n\langle\langle\mathbf{D}(\varepsilon * \mathbf{G} * \cdots * \mathbf{G})\rangle\rangle \\
& =n\langle\langle\mathbf{d}(\varepsilon * \mathbf{G} * \cdots * \mathbf{G})\rangle\rangle
\end{aligned}
$$

using the Bianchi identity and (154). As a consequence

$$
\begin{gathered}
\delta_{\varepsilon} \mathcal{C S}(\mathbf{h})=n \|\left\langle\mathbf { d } \left(\varepsilon * \mathbf{G} * \cdots * \mathbf{G}-\int_{0}^{1} d t t\left(\mathbf{h} * \mathbf{D} \varepsilon * \mathbf{G}_{t}\right.\right.\right. \\
* \cdots * \mathbf{G}_{t}+\mathbf{h} * \mathbf{G}_{t} * \mathbf{D} \varepsilon * \cdots * \mathbf{G}_{t} \\
\left.\left.\left.\left.+\cdots+\mathbf{h} * \mathbf{G}_{t} * \cdots * \mathbf{G}_{t} * \mathbf{D} \varepsilon\right)\right)\right\rangle\right\rangle=0
\end{gathered}
$$

This proves the HS gauge invariance of $\mathcal{C S}(\mathbf{h})$ in a space of odd dimension $d=2 n-1$. 


\subsection{HS consistent anomalies}

Equation (163) gives us the form of the primitive generating functional for consistent anomaly in $d=2 n-2$ dimension:

$$
\begin{aligned}
\mathcal{A}(\mathbf{h}, \varepsilon) & \\
= & n\left\langle\left\langle\varepsilon * \mathbf{G} * \cdots * \mathbf{G}-\int_{0}^{1} d t t\left(\mathbf{h} * \mathbf{D} \varepsilon * \mathbf{G}_{t} * \cdots * \mathbf{G}_{t}\right.\right.\right. \\
& +\mathbf{h} * \mathbf{G}_{t} * \mathbf{D} \varepsilon * \cdots * \mathbf{G}_{t} \\
& \left.\left.+\cdots+\mathbf{h} * \mathbf{G}_{t} * \cdots * \mathbf{G}_{t} * \mathbf{D} \varepsilon\right)\right\rangle .
\end{aligned}
$$

One can write this in a more compact form (see Appendix B):

$$
\begin{aligned}
\mathcal{A}(\mathbf{h}, \varepsilon)= & n \int_{0}^{1} d t(1-t)\left\langle\left\langle\mathbf{h} * \mathbf{d} \varepsilon * \mathbf{G}_{t} * \cdots * \mathbf{G}_{t}\right.\right. \\
& +\mathbf{h} * \mathbf{G}_{t} * \mathbf{d} \varepsilon * \cdots * \mathbf{G}_{t} \\
& \left.\left.+\cdots+\mathbf{h} * \mathbf{G}_{t} * \cdots * \mathbf{G}_{t} * \mathbf{d} \varepsilon\right\rangle\right\rangle
\end{aligned}
$$

where the integrals are over a $4 n-4$ dimensional phase space.

Of course we have to prove that the $\mathcal{A}$ is consistent. The shortest way is to promote $\varepsilon$ to anticommuting parameter (denoting it with the same symbol). So

$s \mathbf{h}=\mathbf{d} \varepsilon-i[\mathbf{h} * \varepsilon], \quad s \varepsilon=\frac{i}{2}[\varepsilon, \varepsilon]=i \varepsilon * \varepsilon, \quad$ etc.

Using these BRST transform one can prove (see Appendix C) that

$s \mathcal{A}(\mathbf{h}, \varepsilon)=0$.

With the familiar manipulations one can rewrite (165) as follows

$$
\begin{gathered}
\mathcal{A}(\mathbf{h}, \varepsilon)=n \int_{0}^{1} d t\left\langle\left\langle\varepsilon * \mathbf{G}_{t} * \cdots * \mathbf{G}_{t}+i(1-t) \varepsilon\right.\right. \\
*\left[\mathbf{h}_{t} * \mathbf{h} * \mathbf{G}_{t} * \cdots * \mathbf{G}_{t}+\mathbf{G}_{t} * \mathbf{h} * \cdots * \mathbf{G}_{t}\right. \\
\left.\left.\left.+\cdots+\mathbf{G}_{t} * \cdots * \mathbf{G}_{t} * \mathbf{h}\right]\right\rangle\right\rangle .
\end{gathered}
$$

The (unintegrated) anomaly is obtained by differentiating with respect to $\varepsilon$ :

$$
\begin{aligned}
\mathcal{A}(\mathbf{h})=n & \int_{0}^{1} d t\left(\mathbf{G}_{t} * \cdots * \mathbf{G}_{t}+i(1-t)\right. \\
& \times\left[\mathbf{h}_{t} * \mathbf{h} * \mathbf{G}_{t} * \cdots * \mathbf{G}_{t}+\mathbf{G}_{t} * \mathbf{h} * \cdots * \mathbf{G}_{t}\right. \\
& \left.\left.\left.+\cdots+\mathbf{G}_{t} * \cdots * \mathbf{G}_{t} * \mathbf{h}\right]\right\rangle\right\rangle
\end{aligned}
$$

which is a $2 n-2=d$ form.

This should be understood as the violation the off shell conservation $\mathcal{D}_{x}^{* \mu} J_{\mu}(x, u)=0$, i.e.

$$
\begin{aligned}
\mathcal{D}_{x}^{* \mu} J_{\mu}(x, u) \sim & \int_{0}^{1} d t \star\left(\mathbf{G}_{t} * \cdots * \mathbf{G}_{t}+i(1-t)\right. \\
& \times\left[\mathbf{h}_{t} * \mathbf{h} * \mathbf{G}_{t} * \cdots * \mathbf{G}_{t}+\mathbf{G}_{t} * \mathbf{h} * \cdots * \mathbf{G}_{t}\right. \\
& \left.\left.+\cdots+\mathbf{G}_{t} * \cdots * \mathbf{G}_{t} * \mathbf{h}\right]\right)
\end{aligned}
$$

where $\star$ denotes the Hodge dual.
Of course the next problem is to understand if and when these anomalies appear. This is not difficult. If the matter fermion model of Sect. 2 is constructed with massless chiral fermions (instead of massive Dirac fermions) it may be anomalous and with the same anomaly coefficient as in the ordinary case. In fact the lowest order term $\mathcal{O}\left(u^{0}\right)$ starts with exactly the same term, but consistency requires an infinite tail of anomalous terms. Moreover (170) implies that there are infinite many conservation laws that are violated, each one with its infinite long anomaly.

Another question is: do these anomalies exhaust the set of possible anomalies in HS theories. We do not know the answer to this question. We know that in ordinary gauge theories the analogous anomalies are the only consistent ones. But the analogy does not seem to be enough to exclude other possible consistent anomalies in HS theories.

\section{Conclusions}

In this paper we have obtained a series of results on HS effective actions derived by integrating out simple (scalar and fermion) matter fields coupled to external HS sources. In particular we have developed methods to compute current correlators, which are the building blocks of the effective action. We have shown how to compute the latter in different ways, either by using the perturbative formalism introduced in [15] or by means of the more traditional Feynman diagrams. The second part of the paper has dealt with interpretations of HS objects, their relations and further developments based on the HS symmetry. In particular we have found a precise relation between the master field $h_{a}(x, u)$ and $h(x, u)$ which couple linearly to fermionic and bosonic matter, respectively. The $h_{a}(x, u)$ potential, with its gauge transformations, lends itself to a simple interpretation as HS gauge field (although the transform contains not only ordinary gauge transformations but also diffeomorphisms, beside other transformations involving all spins). In the third part of the paper we have used this analogy to compute possible obstructions in constructing (even dimensional) HS theories. Such obstructions are the analogue of consistent anomalies in ordinary gauge theories, but, beside the analogy, there are also significant differences. In particular we have seen that, whenever one such an obstruction appears, there are infinite many anomalous currents, and each anomaly consists of an infinite expressions in the local fields. In passing we have shown that one can construct odd dimensional CS actions.

The double nature of $h_{a}(x, u)$ will be at the root of the next two papers, II and III. As a gauge-like object it will be used in paper II to construct HS YM-like theories. As a geometrylike object it will allow us in paper III to interpret the HS geometry within a scheme analogous to teleparallelism. 
Acknowledgements This research has been supported by the Croatian Science Foundation under the Project no. 8946 and by the University of Rijeka under the research support no. 13.12.1.4.05. The research of S.G. has been supported by the Israel Science Foundation (ISF), Grant no. $244 / 17$.

Data Availability Statement This manuscript has no associated data or the data will not be deposited. [Authors' comment: There are no external data associated with the manuscript.]

Open Access This article is distributed under the terms of the Creative Commons Attribution 4.0 International License (http://creativecomm ons.org/licenses/by/4.0/), which permits unrestricted use, distribution, and reproduction in any medium, provided you give appropriate credit to the original author(s) and the source, provide a link to the Creative Commons license, and indicate if changes were made.

Funded by SCOAP ${ }^{3}$.

\section{Appendices}

\section{A Derivation of (31) and (32)}

The Fourier transform of the first line of (25) is elementary.

Let us see next

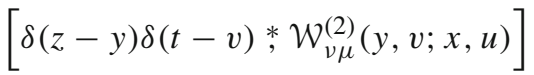

$$
\begin{aligned}
& =i \int \frac{d^{d} r}{(2 \pi)^{d}} \int d^{d} w \\
& \times\left(e^{-i r \cdot(z-y)} e^{i w \cdot(t-v)} e^{\frac{i}{2}\left(\overleftarrow{\left.\partial_{y} \cdot \overrightarrow{\partial_{v}}-\overrightarrow{\partial_{y}} \cdot \overleftarrow{\partial_{v}}\right)}\right.}\left\langle J_{v}(y, v) J_{\mu}(x, u)\right\rangle\right.
\end{aligned}
$$

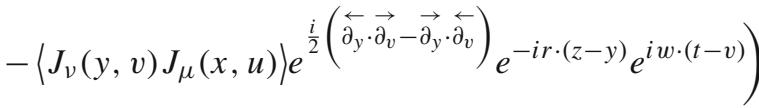

$$
\begin{aligned}
& =i \frac{d^{d} r}{(2 \pi)^{d}} \int d^{d} w e^{-i r \cdot(z-y)} e^{i w \cdot(t-v)} \\
& \times\left(e^{-\frac{1}{2}\left(r \cdot \overrightarrow{\partial_{v}}+w \cdot \overrightarrow{\partial_{y}}\right)}\left\langle J_{v}(y, v) J_{\mu}(x, u)\right\rangle\right. \\
& \left.-\left\langle J_{v}(y, v) J_{\mu}(x, u)\right\rangle e^{\frac{1}{2}\left(r \cdot \overleftarrow{\partial_{v}}+w \cdot \overleftarrow{\partial_{y}}\right)}\right) \\
& =i \frac{d^{d} r}{(2 \pi)^{d}} \int d^{d} w e^{-i r \cdot(z-y)} e^{i w \cdot(t-v)} \\
& \times\left(\left\langle J_{v}\left(y-\frac{w}{2}, v-\frac{r}{2}\right) J_{\mu}(x, u)\right\rangle\right. \\
& \left.-\left\langle J_{v}\left(y+\frac{w}{2}, v+\frac{r}{2}\right) J_{\mu}(x, u)\right\rangle\right) \text {. }
\end{aligned}
$$

The Fourier transform of this is

$$
\begin{aligned}
\int d^{d} x e^{i k_{1} \cdot x} \int d y e^{i k_{2} \cdot y} \int d^{d} z e^{-i q \cdot z} \\
\times\left[\delta(z-y) \delta(t-v) \stackrel{*}{\left.\mathcal{W}_{\nu \mu}^{(2)}(y, v ; x, u)\right]}\right. \\
=i \int d^{d} x e^{i k_{1} \cdot x} \int d y e^{i k_{2} \cdot y} \int d^{d} z e^{-i q \cdot z}
\end{aligned}
$$

$$
\begin{aligned}
& \times \int d^{d} r \int d^{d} w e^{-i r \cdot(z-y)} e^{i w \cdot(t-v)} \\
& \times\left(\left\langle J_{v}\left(y-\frac{w}{2}, v-\frac{r}{2}\right) J_{\mu}(x, u)\right\rangle\right. \\
& \left.-\left\langle J_{v}\left(y+\frac{w}{2}, v+\frac{r}{2}\right) J_{\mu}(x, u)\right\rangle\right) .
\end{aligned}
$$

Integrating over $z$ we find $\delta(q+r)$, and over $w$ we find $\delta\left(t-v-\frac{k_{1}}{2}\right)$ for the first piece and $\delta\left(t-v+\frac{k_{1}}{2}\right)$ for the second. Next, performing the Fourier transform, one finds

$$
\begin{aligned}
\int d^{d} x e^{i k_{1} \cdot x} \int d y e^{i k_{2} \cdot y} \int d^{d} z e^{-i q \cdot z} \\
\times\left[\delta(z-y) \delta(t-v) * \mathcal{W}_{\nu \mu}^{(2)}(y, v ; x, u)\right] \\
=i \delta\left(t-v-\frac{k_{1}}{2}\right)\left\langle\widetilde{J}_{\mu}\left(k_{1}, u\right) \widetilde{J}_{v}\left(-k_{1}, v+\frac{q}{2}\right)\right\rangle \\
-i \delta\left(t-v+\frac{k_{1}}{2}\right)\left\langle\widetilde{J}_{\mu}\left(k_{1}, u\right) \widetilde{J}_{v}\left(-k_{1}, v-\frac{q}{2}\right)\right\rangle .
\end{aligned}
$$

\section{B Explicit calculation of 0- and 1-point function}

\section{B.1 The first term of (19)}

Let us calculate the vacuum energy for fermions. First, the determinant of the inverse propagator can be written as

$\operatorname{det}(-\widehat{P}-m)=\operatorname{det}\left(-\widehat{P}^{2}+m^{2}\right)^{1 / 2}$.

The $\mathcal{W}^{(0)}[h]$ part of the effective action is

$$
\begin{aligned}
\mathcal{W}^{(0)}[h] & =\frac{N}{2} \ln \left[\operatorname{det}\left(-\widehat{P}^{2}+m^{2}\right)\right] \\
& =\frac{N}{2} \operatorname{Tr}\left[\ln \left(-\widehat{P}^{2}+m^{2}\right)\right] \\
& =\frac{N}{2} \int \frac{d^{d} u}{(2 \pi)^{d}} \operatorname{tr}\left(\left\langle u\left|\ln \left(-\widehat{P}^{2}+m^{2}\right)\right| u\right\rangle\right) \\
& =\frac{N}{2} \int \frac{d^{d} u}{(2 \pi)^{d}} \ln \left(-u^{2}+m^{2}\right) \operatorname{tr}(\langle u \mid u\rangle) \\
& =2^{\left\lfloor\frac{d}{2}\right\rfloor-1} N \int \frac{d^{d} u}{(2 \pi)^{d}} \ln \left(-u^{2}+m^{2}\right) \int d^{d} x
\end{aligned}
$$

where we have assumed the symbol of $\widehat{P}$ is $u$. To calculate the integral we make a Wick rotation $u^{0} \rightarrow i u_{E}^{0}$ :

$$
\begin{aligned}
\mathcal{W}^{(0)}[h] & =2^{\left\lfloor\frac{d}{2}\right\rfloor-1} N i \int \frac{d^{d} u_{E}}{(2 \pi)^{d}} \ln \left(u_{E}^{2}+m^{2}\right) \int d^{d} x \\
& =-2^{\left\lfloor\frac{d}{2}\right\rfloor-1-d} N i m^{d} \pi^{-d / 2} \Gamma\left(-\frac{d}{2}\right) \int d^{d} x .
\end{aligned}
$$

This is infinite. Since we are not interested in this paper in cosmological constant problems, we will set it to zero. 


\section{B.2 The second term of (19)}

Let us now focus on the tadpole contribution. The $\mathcal{W}^{(1)}[h]$ part of the effective action is

$\mathcal{W}^{(1)}[h]=\int d^{d} x \int \frac{d^{d} u}{(2 \pi)^{d}} \mathcal{W}_{a}^{(1)}(x, u) h^{a}(x, u)$

where $\mathcal{W}_{a}^{(1)}(x, u)$ is given by (49) and $h^{a}(x, u)$ is defined with (15). Note that the integrals in $\mathcal{W}^{(1)}[h]$ over the odd number of $u$ 's vanish. We are left with:

$$
\begin{aligned}
\mathcal{W}^{(1)}[h]= & -2^{\left\lfloor\frac{d}{2}\right\rfloor} N i \int d^{d} x \int \frac{d^{d} u}{(2 \pi)^{d}} \int_{-\infty}^{\infty} \frac{d \omega}{2 \pi} \frac{e^{i \omega \epsilon}}{\omega} \\
& \times \sum_{n=0}^{\infty} \frac{1}{(2 n+1) !} h_{(2 n+2)}^{a a_{1} \ldots a_{2 n+1}}(x) \frac{u_{a} u_{a_{1}} \ldots u_{a_{2 n+1}}}{u^{2}-m^{2}} .
\end{aligned}
$$

The integral over the $u$ momenta is

$$
\begin{aligned}
\int \frac{d^{d} u}{(2 \pi)^{d}} \frac{u_{a} u_{a_{1}} \ldots u_{a_{2 n+1}}}{u^{2}-m^{\prime 2}} & \\
= & \frac{(2 n+1) ! !}{\prod_{i=0}^{n}(d+2 i)} \eta_{\left(a a_{1}\right.} \ldots \eta_{\left.\mu_{2 n} a_{2 n+1}\right)} \int \frac{d^{d} u}{(2 \pi)^{d}} \frac{u^{2 n+2}}{u^{2}-m^{2}} \\
= & i \frac{(-1)^{n}(2 n+1) ! !}{2^{d+n+1}} \pi^{-d / 2} m^{\prime 2 n+d} \\
& \times \Gamma\left(-n-\frac{d}{2}\right) \eta_{\left(a a_{1}\right.} \ldots \eta_{\left.\mu_{2 n} a_{2 n+1}\right)} .
\end{aligned}
$$

Contracting $\eta$ 's with $h_{(2 n+2)}^{\mu \mu_{1} \ldots \mu_{2 n+1}}$ gives the $(n+1)$ st trace of the spin $(2 n+2)$ field, which we will denote by $h_{(2 n+2)}^{[n+1]}$. Altogether we have

$$
\begin{aligned}
\mathcal{W}^{(1)}[h]= & 2^{\left\lfloor\frac{d}{2}\right\rfloor-d-1} N \pi^{-d / 2} \int d^{d} x \int_{-\infty}^{\infty} \frac{d \omega}{2 \pi} \frac{e^{i \omega \epsilon}}{\omega} \\
& \times \sum_{n=0}^{\infty} \frac{(-1)^{n}(2 n+1) ! !}{2^{n}(2 n+1) !} \Gamma\left(-n-\frac{d}{2}\right) \\
& \times(m-i \omega-\varepsilon)^{2 n+d} h_{(2 n+2)}^{[n+1]}(x) \\
= & 2^{\left\lfloor\frac{d}{2}\right\rfloor-d-2} i N \pi^{-d / 2} \int d^{d} x \sum_{n=0}^{\infty} \frac{(-1)^{n}(2 n+1) ! !}{2^{n}(2 n+1) !} \\
& \times \Gamma\left(-n-\frac{d}{2}\right) m^{2 n+d} h_{(2 n+2)}^{[n+1]}(x) .
\end{aligned}
$$

Finally, the effective action up to first order in $h$ is

$$
\begin{aligned}
\mathcal{W}[h] \approx & -2^{\left\lfloor\frac{d}{2}\right\rfloor-1-d} N i m^{d} \pi^{-d / 2} \\
& \times \Gamma\left(-\frac{d}{2}\right) \int d^{d} x\left(1-\frac{1}{2} h_{(2)}^{[1]}(x)\right) \\
& +2^{\left\lfloor\frac{d}{2}\right\rfloor-d-2} i N \pi^{-d / 2} \int d^{d} x \\
& \times \sum_{n=1}^{\infty} \frac{(-1)^{n}(2 n+1) ! ! \Gamma\left(-n-\frac{d}{2}\right)}{2^{n}(2 n+1) !} m^{2 n+d} h_{(2 n+2)}^{[n+1]}(x)
\end{aligned}
$$

$$
+\cdots
$$

One would expect that the first line in (181) corresponds to the cosmological constant term which is of the form

$\Lambda \int d^{d} x \sqrt{g} \sim \Lambda \int d^{d} x\left(1+\frac{1}{2} h_{(2)}^{[1]}(x)+\ldots\right)$.

We notice that there is a minus sign next to $h_{(2)}^{[1]}(x)$ term in the first line of (181), instead of plus. On the other hand this result is infinite for even $d$, and therefore scheme dependent. Since in this paper we are interested in flat spacetime we will assume that the first line of (181) can be renormalized to 0 .

\section{Gauge transformations in the bosonic model}

In this appendix we present the general form of the gauge transformations in the bosonic model. In order to keep the formulae as simple as possible, for the time being we define the $*$-product as

$\alpha(x, u) * \beta(x, u)=\alpha(x, u) e^{i\left(\overleftarrow{\partial}_{x} \cdot \vec{\partial}_{u}-\vec{\partial}_{x} \cdot \overleftarrow{\partial}_{u}\right)} \beta(x, u)$

We consider first the general case

$$
\begin{aligned}
& h(x, u)=\sum_{p=0}^{s^{\prime}} \frac{1}{p !} \stackrel{(p)}{h}^{\mu_{1} \ldots \mu_{p}} u_{\mu_{1}} \ldots u_{\mu_{p}} \\
& \eta(x, u)=\frac{1}{(s-1) !} \stackrel{(s-1)}{\epsilon}^{\mu_{1} \ldots \mu_{s-1}} u_{\mu_{1}} \ldots u_{\mu_{s-1}} . \\
& \delta_{\epsilon}^{(1)} h(x, u)=-\frac{i}{2}[h(x, u) \stackrel{*}{,} \epsilon(x, u)] \\
& =h(x, u) \sin \left(\overleftarrow{\partial}_{x} \cdot \vec{\partial}_{u}-\vec{\partial}_{x} \cdot \overleftarrow{\partial}_{u}\right) \epsilon(x, u) \\
& =\sum_{r=0}^{\left[\frac{s+s^{\prime}-2}{2}\right]} \frac{(-)^{r}}{(2 r+1) !} h(x, u) \\
& \times\left(\overleftarrow{\partial}_{x} \cdot \vec{\partial}_{u}-\vec{\partial}_{x} \cdot \overleftarrow{\partial}_{u}\right)^{2 r+1} \epsilon(x, u) \\
& =\sum_{r=0}^{\left[\frac{s+s^{\prime}-2}{2}\right]} \sum_{k=0}^{2 r+1} \frac{(-)^{r-k+1}}{(2 r+1) !}\left(\begin{array}{c}
2 r+1 \\
k
\end{array}\right) h(x, u) \\
& \times\left(\overleftarrow{\partial}_{x} \cdot \vec{\partial}_{u}\right)^{k}\left(\vec{\partial}_{x} \cdot \overleftarrow{\partial}_{u}\right)^{2 r+1-k} \epsilon(x, u) \\
& =\sum_{r=0}^{\left[\frac{s^{\prime}-1}{2}\right]} \frac{(-)^{r+1}}{(2 r+1) !} h(x, u)\left(\vec{\partial}_{x} \cdot \overleftarrow{\partial}_{u}\right)^{2 r+1} \epsilon(x, u) \\
& +\sum_{k=1}^{s-1} \sum_{r=\left[\frac{k}{2}\right]}^{\left[\frac{k-1+s^{\prime}}{2}\right]} \frac{(-)^{r-k+1}}{(2 r+1) !}\left(\begin{array}{c}
2 r+1 \\
k
\end{array}\right)
\end{aligned}
$$




$$
\begin{aligned}
& \times h(x, u)\left(\overleftarrow{\partial}_{x} \cdot \vec{\partial}_{u}\right)^{k}\left(\vec{\partial}_{x} \cdot \overleftarrow{\partial}_{u}\right)^{2 r+1-k} \epsilon(x, u) \\
= & \sum_{k=0}^{s-1} \sum_{r=\left[\frac{k}{2}\right]}^{\left.\frac{k-1+s^{\prime}}{2}\right]} \frac{(-)^{r-k+1}}{(2 r+1) !}\left(\begin{array}{c}
2 r+1 \\
k
\end{array}\right) \\
& \times h(x, u)\left(\overleftarrow{\partial}_{x} \cdot \vec{\partial}_{u}\right)^{k}\left(\vec{\partial}_{x} \cdot \overleftarrow{\partial}_{u}\right)^{2 r+1-k} \epsilon(x, u)
\end{aligned}
$$

The condition $0 \leq r \leq\left[\frac{s^{\prime}-1}{2}\right]$ for the first term comes from the requirement $k=0$ and $2 r+1 \leq s^{\prime}$. The bounds on $k$ in the second term come from $k \leq s-1$. The bounds on $r$ come from the condition $k \leq 2 r+1$, i.e. $r \geq\left[\frac{k}{2}\right]$ and $2 r+1-k \leq s^{\prime}$,i.e. $r \leq\left[\frac{k-1+s^{\prime}}{2}\right]$.

$$
\begin{aligned}
& \frac{\partial}{\partial u_{\mu_{1}}} \cdots \frac{\partial}{\partial u_{\mu_{t}}} \sum_{k=0}^{s-1} \sum_{r=\left[\frac{k}{2}\right]}^{\left.\frac{k-1+s^{\prime}}{2}\right]} \frac{(-)^{r-k+1}}{(2 r+1) !}\left(\begin{array}{c}
2 r+1 \\
k
\end{array}\right) \\
& \times\left. h(x, u)\left(\overleftarrow{\partial}_{x} \cdot \vec{\partial}_{u}\right)^{k}\left(\vec{\partial}_{x} \cdot \overleftarrow{\partial}_{u}\right)^{2 r+1-k} \epsilon(x, u)\right|_{u=0} \\
& =\sum_{k=0}^{s-1} \sum_{r=\left[\frac{k}{2}\right]}^{\left[\frac{k-1+s^{\prime}}{2}\right]} \frac{(-)^{r-k+1}}{(2 r+1) !}\left(\begin{array}{c}
2 r+1 \\
k
\end{array}\right) \sum_{i=0}^{t}\left(\begin{array}{c}
t \\
t-i
\end{array}\right) \\
& \frac{\partial}{\partial u_{\left(\mu_{i+1}\right.}} \ldots \frac{\partial}{\partial u_{\mu_{t}}} h(x, u)\left(\overleftarrow{\partial}_{x} \cdot \vec{\partial}_{u}\right)^{k}\left(\vec{\partial}_{x} \cdot \overleftarrow{\partial}_{u}\right)^{2 r+1-k} \\
& \times\left.\frac{\partial}{\partial u_{\mu_{1}}} \ldots \frac{\partial}{\partial u_{\left.\mu_{i}\right)}} \epsilon(x, u)\right|_{u=0} \\
& =\sum_{k=0}^{s-1} \sum_{r=\left[\frac{k}{2}\right]}^{\left[\frac{k-1+s^{\prime}}{2}\right]} \frac{(-)^{r-k+1}}{(2 r+1) !}\left(\begin{array}{c}
2 r+1 \\
k
\end{array}\right) \sum_{i=0}^{t}\left(\begin{array}{c}
t \\
t-i
\end{array}\right) \\
& \partial_{\nu_{1}} \ldots \partial_{\nu_{k}}{ }^{(t+2 r+1-k-i)^{\nu_{k+1} \ldots \nu_{2 r+1}\left(\mu_{i+1} \ldots \mu_{t}\right.}} h \\
& \times \partial_{\nu_{k+1}} \ldots \partial_{\nu_{2 r+1}} \stackrel{(k+i)}{\epsilon}^{\left.\left|\nu_{1} \ldots \nu_{k}\right| \mu_{1} \ldots \mu_{i}\right)}
\end{aligned}
$$

This implies the constraints $i+k=s-1$ and $t+2 r+$ $1-k-i \leq s^{\prime}$, implying $t \leq s+s^{\prime}-2$. Therefore $0 \leq i=$ $s-k-1 \leq t$ which means $s-t-1 \leq k \leq s-1$. If $t \leq s-2$, this is the most restrictive condition; if $s-1 \leq t \leq s+s^{\prime}-2$ then $0 \leq k \leq s-1$. On the other hand we have $2 r+1 \geq$ $k \geq s-t-1$,i.e. $r \geq\left[\frac{k}{2}\right]$ and $t+2 r+1-k-i \leq s$,i.e. $2 r+1 \leq s+s^{\prime}-t-1 \leq s^{\prime}+k$.

So, for $t \leq s-2$,

$$
\begin{aligned}
& \sum_{k=s-t-1}^{s-1} \sum_{r=\left[\frac{k}{2}\right]}^{\left[\frac{s+s^{\prime}-t-2}{2}\right]} \frac{(-)^{r-k+1}}{(2 r+1) !}\left(\begin{array}{c}
2 r+1 \\
k
\end{array}\right)\left(\begin{array}{c}
t \\
s-k-1
\end{array}\right) \\
& \partial_{\nu_{1}} \ldots \partial_{\nu_{k}}{ }^{(t-s+1+2 r+1)^{\nu_{k+1} \ldots \nu_{2 r+1}}\left(\mu_{s} \ldots \mu_{t+k}\right.} \\
& \times \partial_{\nu_{k+1}} \ldots \partial_{\nu_{2 r+1}}(s-1)^{\left.\left|\nu_{1} \ldots \nu_{k}\right| \mu_{k+1} \ldots \mu_{s-1}\right)}
\end{aligned}
$$

For $s-1 \leq t \leq s+s^{\prime}-2$,

$$
\begin{aligned}
& \sum_{k=0}^{s-1} \sum_{r=\left[\frac{k}{2}\right]}^{\left.\frac{s+s^{\prime}-t-2}{2}\right]} \frac{(-)^{r-k+1}}{(2 r+1) !}\left(\begin{array}{c}
2 r+1 \\
k
\end{array}\right)\left(\begin{array}{c}
t \\
s-k-1
\end{array}\right) \\
& \partial_{\nu_{1}} \ldots \partial_{\nu_{k}}{ }^{(t-s+1+2 r+1)^{\nu_{k+1} \ldots \nu_{2 r+1}\left(\mu_{s} \ldots \mu_{t+k}\right.} h}
\end{aligned}
$$

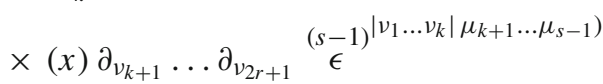

$$
\begin{aligned}
& \text { All } \delta \stackrel{(t)}{h} \quad \text { with } t \geq s+s^{\prime}-1 \text { are vanishing. }
\end{aligned}
$$

\section{C.1 Truncated transformations}

Let us work out some examples explicitly. For $s=1$ and $s^{\prime}=3$ we get

$$
\begin{aligned}
& \delta \stackrel{(0)}{h}=\delta^{(1)} \stackrel{(0)}{h} \\
& =-\stackrel{(1)^{\nu_{1}}}{h} \partial_{\nu_{1}} \epsilon+\frac{1}{6} \stackrel{(3)}{h}^{\nu_{1} \nu_{2} \nu_{3}} \partial_{\nu_{1}} \partial_{\nu_{2}} \partial_{\nu_{3}} \epsilon \\
& \delta \stackrel{(1)}{h}^{\mu_{1}}=\delta^{(0)} \stackrel{(1)^{\mu_{1}}}{h}+\delta^{(1)} \stackrel{(1)^{\mu_{1}}}{h} \\
& =\partial^{\mu_{1}} \epsilon-\stackrel{(2)^{\nu_{1} \mu_{1}}}{h} \partial_{\nu_{1}} \epsilon \\
& \delta \stackrel{(2)}{h}^{\mu_{1} \mu_{2}}=\delta^{(1)} \stackrel{(1)}{h}^{\mu_{1} \mu_{2}} \\
& \text { (3) } v_{1} \mu_{1} \mu_{2} \\
& =-h \quad \partial_{\nu_{1}} \epsilon \\
& \delta h^{(3)^{\mu_{1} \mu_{2} \mu_{3}}}=0 \\
& \because::
\end{aligned}
$$

For $s=2$ and $s^{\prime}=3$ we get

$$
\begin{aligned}
& \delta \stackrel{(0)}{h}=\delta^{(1)} \stackrel{(0)}{h} \\
& =\epsilon^{\nu_{1}} \partial_{\nu_{1}} \stackrel{(0)}{h}-\frac{1}{2} \partial_{\nu_{1}} \stackrel{(2)^{\nu_{2} \nu_{3}}}{h} \partial_{\nu_{2}} \partial_{\nu_{3}} \epsilon^{\nu_{1}} \\
& \delta \stackrel{(1)}{h}^{\mu_{1}}=\delta^{(1)} \stackrel{(1)}{h}^{\mu_{1}} \\
& =\epsilon^{\nu_{1}} \partial_{\nu_{1}} \stackrel{(1)^{\mu_{1}}}{h}-\stackrel{(1)^{\nu_{1}}}{h} \partial_{\nu_{1}} \epsilon^{\mu_{1}} \\
& +\frac{1}{6} \stackrel{(3)}{h}^{\nu_{1} \nu_{2} \nu_{3}} \partial_{\nu_{1}} \partial_{\nu_{2}} \partial_{\nu_{3}} \epsilon^{\mu_{1}} \\
& -\frac{1}{2} \partial_{\nu_{1}} \stackrel{(3)^{\nu_{2} \nu_{3} \mu_{1}}}{{ }^{2}} \partial_{\nu_{2}} \partial_{\nu_{3}} \epsilon^{\nu_{1}} \\
& \delta \stackrel{(2)}{h}^{\mu_{1} \mu_{2}}=\delta^{(0)} \stackrel{(2)}{h}{ }^{\mu_{1} \mu_{2}}+\delta^{(1)} \stackrel{(2)}{h}^{\mu_{1} \mu_{2}} \\
& =2 \partial^{\left(\mu_{1} \mid\right.} \epsilon^{\left.\mu_{2}\right)} \\
& +\epsilon^{\nu_{1}} \partial_{\nu_{1}} \stackrel{(2)}{h}^{\mu_{1} \mu_{2}}-2 h^{(2)^{\nu_{1}\left(\mu_{1}\right.}} \partial_{\nu_{1}} \epsilon^{\left.\mu_{2}\right)} \\
& \delta \stackrel{(3)}{h}^{\mu_{1} \mu_{2} \mu_{3}}=\epsilon^{\nu_{1}} \partial_{\nu_{1}} \stackrel{(3)}{h}^{\mu_{1} \mu_{2} \mu_{3}}-3 \stackrel{(3)}{h}^{\nu_{1}\left(\mu_{1} \mu_{2}\right.} \partial_{\nu_{1}} \epsilon^{\left.\mu_{3}\right)} \\
& \delta \stackrel{(4)}{h}^{\mu_{1} \mu_{2} \mu_{3} \mu_{4}}=0
\end{aligned}
$$




$$
\vdots: \vdots
$$

For $s=3$ and $s^{\prime}=3$ we get

$$
\begin{aligned}
& \stackrel{(0)}{h}=\delta^{(1)} \stackrel{(0)}{h} \\
& =\frac{1}{2} \partial_{\nu_{1}} \partial_{\nu_{2}} \stackrel{(1)^{\nu_{3}}}{h} \partial_{\nu_{3}} \epsilon^{\nu_{1} \nu_{2}} \\
& -\frac{1}{12} \partial_{\nu_{1}} \partial_{\nu_{2}} \stackrel{(3)^{\nu_{3} \nu_{4} \nu_{5}}}{h} \partial_{\nu_{3}} \partial_{\nu_{4}} \partial_{5} \epsilon^{\nu_{1} \nu_{2}} \\
& \delta \stackrel{(1)}{h}^{\mu_{1}}=\delta^{(1)} \stackrel{(1)}{h}^{\mu_{1}} \\
& =\epsilon^{\nu_{1} \mu_{1}} \partial_{\nu_{1}} \stackrel{(0)}{h} \\
& -\frac{1}{2} \partial_{\nu_{1}} \stackrel{(2)}{h}^{\nu_{2} \nu_{3}} \partial_{\nu_{2}} \partial_{\nu_{3}} \epsilon^{\nu_{1} \mu_{1}} \\
& +\frac{1}{2} \partial_{\nu_{1}} \partial_{\nu_{2}} \stackrel{(2)}{h}^{\nu_{3} \mu_{1}} \partial_{\nu_{3}} \epsilon^{\nu_{1} \nu_{2}} \\
& \delta \stackrel{(2)^{\mu_{1} \mu_{2}}}{h}=\delta^{(1)} \stackrel{(2)^{\mu_{1} \mu_{2}}}{h} \\
& =2 \epsilon^{\nu_{1}\left(\mu_{1}\right.} \partial_{\nu_{1}} \stackrel{(1)}{h}^{\left.\mu_{2}\right)}-\stackrel{(1)^{\nu_{1}}}{h} \partial_{\nu_{1}} \epsilon^{\mu_{1} \mu_{2}}
\end{aligned}
$$

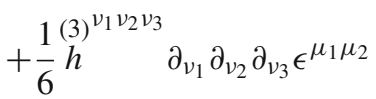

$$
\begin{aligned}
& -\partial_{\nu_{1}} \stackrel{(3)}{h}^{\nu_{2} \nu_{3}\left(\mu_{1}\right.} \partial_{\nu_{2}} \partial_{\nu_{3}} \epsilon^{\left.\left|\nu_{1}\right| \mu_{2}\right)} \\
& +\frac{1}{2} \partial_{\nu_{1}} \partial_{\nu_{2}} \stackrel{(3)^{\nu_{3} \mu_{1} \mu_{2}}}{h} \partial_{\nu_{3}} \epsilon^{\nu_{1} \nu_{2}} \\
& \delta \stackrel{(3)}{h} \stackrel{\mu_{1} \mu_{2} \mu_{3}}{h}=\delta^{(0)} \stackrel{(3)}{h}{ }^{\mu_{1} \mu_{2} \mu_{3}}+\delta^{(1)} \stackrel{(3)}{h}^{\mu_{1} \mu_{2} \mu_{3}} \\
& =3 \partial^{\left(\mu_{1}\right.} \epsilon^{\left.\mu_{2} \mu_{3}\right)} \\
& +3 \epsilon^{\nu_{1}\left(\mu_{1}\right.} \partial_{\nu_{1}} \stackrel{(2)}{h}^{\left.\mu_{2} \mu_{3}\right)} \\
& -3 \stackrel{(2)}{h}^{\nu_{1}\left(\mu_{1}\right.} \partial_{\nu_{1}} \epsilon^{\left.\mu_{2} \mu_{3}\right)} \\
& \delta \stackrel{(4)^{\mu_{1} \mu_{2} \mu_{3} \mu_{4}}}{h}=\delta^{(1)} \stackrel{(4)^{\mu_{1} \mu_{2} \mu_{3} \mu_{4}}}{h} \\
& =4 \epsilon^{\nu_{1}\left(\mu_{1}\right.} \partial_{\nu_{1}} \stackrel{(3)}{h}^{\left.\mu_{1} \mu_{2} \mu_{3}\right)}
\end{aligned}
$$

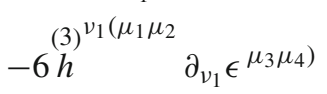

$$
\begin{aligned}
& \delta \stackrel{(5)}{h}^{\mu_{1} \mu_{2} \mu_{3} \mu_{4} \mu_{5}}=0 \\
& \therefore \text {. }
\end{aligned}
$$

\section{Some proofs}

D.1 Proof of Eq. (161)

$$
\begin{aligned}
\delta_{\varepsilon} \mathcal{C S}(\mathbf{h}) & \\
= & n \int_{0}^{1} d t\left(\left\langle\left\langle\mathbf{D} \varepsilon * \mathbf{G}_{t} * \cdots * \mathbf{G}_{t}\right\rangle\right\rangle+t\left\langle\left\langle\mathbf{h} * \mathbf{d}_{t} \mathbf{D} \varepsilon * \cdots * \mathbf{G}_{t}\right\rangle\right\rangle+\cdots\right. \\
& \left.\cdots+t\left\langle\left\langle\mathbf{h} * \mathbf{G}_{t} * \cdots * \mathbf{d}_{t} \mathbf{D} \varepsilon\right\rangle\right\rangle\right)
\end{aligned}
$$

$$
\begin{aligned}
= & n \int_{0}^{1} d t\left(\left\langle\left\langle\mathbf{D} \varepsilon * \mathbf{G}_{t} * \cdots * \mathbf{G}_{t}\right\rangle\right\rangle+t\left\langle\left\langle\mathbf{h} * \mathbf{d}_{t}\left(\mathbf{D} \varepsilon * \cdots * \mathbf{G}_{t}\right)\right\rangle\right\rangle+\cdots\right. \\
& \cdots+t\left\langle\left\langle\mathbf{h} * \mathbf{d}_{t}\left(\mathbf{G}_{t} * \cdots * \mathbf{D} \varepsilon\right)\right\rangle\right) \\
= & n \int_{0}^{1} d t\left(\left\langle\left\langle\mathbf{D} \varepsilon * \mathbf{G}_{t} * \cdots * \mathbf{G}_{t}\right\rangle\right\rangle+t\left\langle\left\langle\frac{d \mathbf{G}_{t}}{d t} * \mathbf{D} \varepsilon * \cdots * \mathbf{G}_{t}\right\rangle\right\rangle+\cdots\right. \\
& \cdots+t\left\langle\left\langle\frac{d \mathbf{G}_{t}}{d t} * \mathbf{G}_{t} * \cdots * \mathbf{D} \varepsilon\right\rangle\right) \\
& -t\left\langle\left\langle\mathbf { d } \left(\mathbf{h} * \mathbf{D} \varepsilon * \mathbf{G}_{t} * \cdots * \mathbf{G}_{t}+\mathbf{h} * \mathbf{G}_{t} * \mathbf{D} \varepsilon * \cdots * \mathbf{G}_{t}+\cdots\right.\right.\right. \\
& \left.\left.\left.\cdots+\mathbf{h} * \mathbf{G}_{t} * \cdots * \mathbf{G}_{t} * \mathbf{D} \varepsilon\right)\right\rangle\right\rangle \\
= & n \int_{0}^{1} d t\left\{\left(\left\langle\mathbf{D} \varepsilon * \mathbf{G}_{t} * \cdots * \mathbf{G}_{t}\right\rangle\right\rangle+t \frac{d}{d t}\left\langle\left\langle\mathbf{D} \varepsilon * \mathbf{G}_{t} * \cdots * \mathbf{G}_{t}\right\rangle\right\rangle\right) \\
& -t\left\langle\left\langle\mathbf { d } \left(\mathbf{h} * \mathbf{D} \varepsilon * \mathbf{G}_{t} * \cdots * \mathbf{G}_{t}+\mathbf{h} * \mathbf{G}_{t} * \mathbf{D} \varepsilon * \cdots * \mathbf{G}_{t}+\cdots\right.\right.\right. \\
& \left.\left.\left.\left.\cdots+\mathbf{h} * \mathbf{G}_{t} * \cdots * \mathbf{G}_{t} * \mathbf{D} \varepsilon\right)\right\rangle\right\rangle\right\} .
\end{aligned}
$$

\section{E From Eqs. (164) to (165)}

The starting point is

$$
\begin{aligned}
\mathcal{A}(\mathbf{h}, \varepsilon)= & n\left\langle\left\langle\varepsilon * \mathbf{G} * \cdots * \mathbf{G}-\int_{0}^{1} d t t\left(\mathbf{h} * \mathbf{D} \varepsilon * \mathbf{G}_{t} * \cdots\right.\right.\right. \\
& * \mathbf{G}_{t}+\mathbf{h} * \mathbf{G}_{t} * \mathbf{D} \varepsilon * \cdots * \mathbf{G}_{t} \\
& \left.\left.\left.+\cdots+\mathbf{h} * \mathbf{G}_{t} * \cdots * \mathbf{G}_{t} * \mathbf{D} \varepsilon\right)\right\rangle\right\rangle .
\end{aligned}
$$

Let us consider the term in round bracket and decompose $\mathbf{D} \varepsilon=\mathbf{d} \varepsilon-i[\mathbf{h}, \varepsilon]$. We consider first the second term, i.e.

$$
\begin{aligned}
& -i t\left\langle\mathbf{h} *[\mathbf{h} * \varepsilon] * \mathbf{G}_{t} * \cdots * \mathbf{G}_{t}+\mathbf{h} * \mathbf{G}_{t} *[\mathbf{h} * \varepsilon] * \cdots\right. \\
& \left.\left.* \mathbf{G}_{t}+\cdots+\mathbf{h} * \mathbf{G}_{t} * \cdots * \mathbf{G}_{t} *[\mathbf{h} * \varepsilon]\right)\right\rangle .
\end{aligned}
$$

Using (154) this becomes

$$
\begin{aligned}
& -i\left\langle\left[\mathbf{h} * \mathbf{h}_{t}\right] * \varepsilon * \mathbf{G}_{t} * \cdots * \mathbf{G}_{t}+\mathbf{h} * \varepsilon *\left[\mathbf{G}_{t} * \mathbf{h}_{t}\right] * \cdots * \mathbf{G}_{t}\right. \\
& \left.\left.\left.\quad+\cdots+\mathbf{h} * \varepsilon * \cdots * \mathbf{G}_{t} *\left[\mathbf{G}_{t} * \mathbf{h}_{t}\right]\right)\right\rangle\right\rangle \\
& -i\left\langle\left[\mathbf{h} * \mathbf{h}_{t}\right] * \mathbf{G}_{t} * \varepsilon \cdots * \mathbf{G}_{t}+\mathbf{h} *\left[\mathbf{G}_{t} * \mathbf{h}_{t}\right] * \varepsilon * \cdots * \mathbf{G}_{t}\right. \\
& \left.\left.\quad+\cdots+\mathbf{h} * \mathbf{G}_{t} * \varepsilon * \cdots * \mathbf{G}_{t} *\left[\mathbf{G}_{t} * \mathbf{h}_{t}\right]\right)\right\rangle \\
& \quad \ldots \\
& \quad-i\left\langle\left[\mathbf{h} * \mathbf{h}_{t}\right] * \mathbf{G}_{t} * \cdots * \mathbf{G}_{t} * \varepsilon+\mathbf{h} *\left[\mathbf{G}_{t} * \mathbf{h}_{t}\right] * * \cdots * \mathbf{G}_{t} * \varepsilon\right. \\
& \left.\left.\quad+\cdots+\mathbf{h} * \mathbf{G}_{t} * \cdots * \mathbf{G}_{t} *\left[\mathbf{G}_{t}, \mathbf{h}_{t}\right] * \varepsilon\right)\right\rangle .
\end{aligned}
$$

Now we use $-i\left[\mathbf{h}_{t}, \mathbf{h}\right]=\frac{d \mathbf{G}_{t}}{d t}-\mathbf{d h}$ and $\left[\mathbf{h}_{t}, \mathbf{G}_{t}\right]=-i \mathbf{d} \mathbf{G}_{t}$ and (185) becomes

$$
\begin{aligned}
& \|\left\langle\frac { d \mathbf { G } _ { t } } { d t } * \left(\varepsilon * \mathbf{G}_{t} * \cdots * \mathbf{G}_{t}+\mathbf{G}_{t} * \varepsilon \cdots * \mathbf{G}_{t}\right.\right. \\
& \left.\left.\quad+\cdots+\mathbf{G}_{t} * \cdots * \mathbf{G}_{t} * \varepsilon\right)\right\rangle \\
& \quad-\left\langle\left\langle\mathbf { d h } * \left(\varepsilon * \mathbf{G}_{t} * \cdots * \mathbf{G}_{t}+\mathbf{G}_{t} * \varepsilon \cdots * \mathbf{G}_{t}\right.\right.\right. \\
& \left.\left.\left.\quad+\cdots+\mathbf{G}_{t} * \cdots * \mathbf{G}_{t} * \varepsilon\right)\right\rangle\right\rangle \\
& \quad+\left\langle\left\langle\mathbf{h} * \varepsilon * \mathbf{d}\left(\mathbf{G}_{t} * \cdots * \mathbf{G}_{t}\right)+\mathbf{h} * \mathbf{d}\left(\mathbf{G}_{t} * \check{\varepsilon} * \cdots * \mathbf{G}_{t}\right)\right.\right. \\
& \left.\left.\quad+\cdots+\mathbf{h} * \mathbf{d}\left(\mathbf{G}_{t} * \cdots * \mathbf{G}_{t}\right) * \varepsilon\right\rangle\right\rangle
\end{aligned}
$$


where over $\varepsilon$ means that $\mathbf{d}$ does not act on it. This is

$$
\begin{aligned}
\frac{d}{d t}\langle\langle\varepsilon & \left.\left.* \mathbf{G}_{t} * \cdots * \mathbf{G}_{t}\right\rangle\right\rangle \\
& -\left\langle\left\langle\mathbf{h} * \mathbf{d} \varepsilon * \mathbf{G}_{t} * \cdots * \mathbf{G}_{t}+\mathbf{h} * \mathbf{G}_{t} * \mathbf{d} \varepsilon * \cdots * \mathbf{G}_{t}\right.\right. \\
& \left.\left.+\cdots+\mathbf{h} * \mathbf{G}_{t} \cdots * \mathbf{G}_{t} * \mathbf{d} \varepsilon\right\rangle\right\rangle \\
& -\left\langle\left\langle\mathbf { d } \left(\mathbf{h} * \varepsilon * \mathbf{G}_{t} * \cdots * \mathbf{G}_{t}+\mathbf{h} * \mathbf{G}_{t} * \varepsilon * \cdots * \mathbf{G}_{t}\right.\right.\right. \\
& \left.\left.+\cdots+\mathbf{h} * \mathbf{G}_{t} \cdots * \mathbf{G}_{t} * \varepsilon\right)\right\rangle .
\end{aligned}
$$

The last line vanishes. Disregarding it and adding the first two lines (under $-\int_{0}^{1} d t$ ) to the remaining piece in (185), i.e.

$$
\begin{aligned}
& \| \varepsilon * \mathbf{G} * \cdots * \mathbf{G}-\int_{0}^{1} d t t\left(\mathbf{h} * \mathbf{d} \varepsilon * \mathbf{G}_{t} * \cdots * \mathbf{G}_{t}\right. \\
& \quad+\mathbf{h} * \mathbf{G}_{t} * \mathbf{d} \varepsilon * \cdots * \mathbf{G}_{t} \\
& \left.\left.\quad+\cdots+\mathbf{h} * \mathbf{G}_{t} * \cdots * \mathbf{G}_{t} * \mathbf{d} \varepsilon\right)\right\rangle
\end{aligned}
$$

and integrating by parts in $t$ the first line of (188), we get

$$
\begin{aligned}
\mathcal{A}(\mathbf{h}, \varepsilon)= & n \int_{0}^{1} d t(1-t)\left\langle\left\langle\mathbf{h} * \mathbf{d} \varepsilon * \mathbf{G}_{t} * \cdots * \mathbf{G}_{t}\right.\right. \\
& +\mathbf{h} * \mathbf{G}_{t} * \mathbf{d} \varepsilon * \cdots * \mathbf{G}_{t} \\
& \left.\left.+\cdots+\mathbf{h} * \mathbf{G}_{t} * \cdots * \mathbf{G}_{t} * \mathbf{d} \varepsilon\right\rangle\right\rangle .
\end{aligned}
$$

\section{F Proof of Eq. (167)}

We prove Eq. (167) for the case $n=3$. In that case

$$
\begin{aligned}
\mathcal{A}(\mathbf{h}, \varepsilon) & =3 \int_{0}^{1} d t(1-t)\left\langle\left\langle\mathbf{h} * \mathbf{d} \varepsilon * \mathbf{G}_{t}+\mathbf{h} * \mathbf{G}_{t} * \mathbf{d} \varepsilon\right\rangle\right\rangle \\
& =+\frac{1}{2}\langle\langle\mathbf{d} \varepsilon *(\mathbf{d h} * \mathbf{h}+\mathbf{h} * \mathbf{d h})\rangle\rangle-\frac{i}{2}\langle\langle\mathbf{d} \varepsilon * \mathbf{h} * \mathbf{h} * \mathbf{h}\rangle\rangle
\end{aligned}
$$

Applying the BRST operator $s$

$$
\begin{aligned}
s \mathcal{A}(\mathbf{h}, \varepsilon)= & +\frac{i}{2}\langle\langle\mathbf{d}(\varepsilon * \varepsilon) *(\mathbf{d h} * \mathbf{h}+\mathbf{h} * \mathbf{d h}) \\
& +\mathbf{d} \varepsilon *([\mathbf{d h} * \varepsilon]-[\mathbf{h} * \mathbf{d} \varepsilon]) * \mathbf{h} \\
& +\mathbf{d} \varepsilon * \mathbf{h} *([\mathbf{d h} * \varepsilon]-[\mathbf{h} * \mathbf{d} \varepsilon])\rangle\rangle \\
& -\frac{1}{2}\langle\langle\mathbf{d} \varepsilon * \mathbf{d h} *(\mathbf{d} \varepsilon-i[\mathbf{h} * \varepsilon]) \\
& +\mathbf{d} \varepsilon *(\mathbf{d} \varepsilon-i[\mathbf{h} * \varepsilon]) * \mathbf{d h}\rangle\rangle \\
& +\frac{1}{2}\langle\langle\mathbf{d}(\varepsilon * \varepsilon) * \mathbf{h} * \mathbf{h} * \mathbf{h}\rangle\rangle \\
& +\frac{i}{2}\langle\langle\mathbf{d} \varepsilon(\mathbf{d} \varepsilon-i[\mathbf{h} * \varepsilon]) * \mathbf{h} * \mathbf{h} \\
& +\mathbf{d} \varepsilon * \mathbf{h} *(\mathbf{d} \varepsilon-i[\mathbf{h} * \varepsilon]) * \mathbf{h} \\
& +\mathbf{d} \varepsilon * \mathbf{h} * \mathbf{h} *(\mathbf{d} \varepsilon-i[\mathbf{h} * \varepsilon])\rangle .
\end{aligned}
$$

Expanding the RHS one can see each term cancels a similar term with opposite sign, except

$\langle\langle\mathbf{d} \varepsilon * \mathbf{d} \varepsilon * \mathbf{d h}\rangle\rangle=\langle\langle\mathbf{d}(\varepsilon * \mathbf{d} \varepsilon * \mathbf{d h})\rangle\rangle=0$

which vanishes by integration, and

$\langle\langle\mathbf{d} \varepsilon * \mathbf{h} * \mathbf{d} \varepsilon * \mathbf{h}\rangle\rangle=-\langle\langle\mathbf{d} \varepsilon * \mathbf{h} * \mathbf{d} \varepsilon * \mathbf{h}\rangle\rangle$

which vanishes by symmetry.

\section{References}

1. X.O. Camanho, J.D. Edelstein, J. Maldacena, A. Zhiboedov, Causality constraints on corrections to the graviton three-point coupling. JHEP 1602, 020 (2016). arXiv:1407.5597 [hep-th]

2. M.A. Vasiliev, Consistent equation for interacting gauge fields of all spins in (3+1)-dimensions. Phys. Lett. B 243, 378 (1990)

3. M.A. Vasiliev, Properties of equations of motion of interacting gauge fields of all spins in (3+1)-dimensions. Class. Quantum Gravity 8, 1387 (1991)

4. M.A. Vasiliev, Algebraic aspects of the higher spin problem. Phys. Lett. B 257, 111 (1991)

5. M.A. Vasiliev, More on equations of motion for interacting massless fields of all spins in (3+1)-dimensions. Phys. Lett. B 285, 225 (1992)

6. D. Francia, A. Sagnotti, On the geometry of higher spin gauge fields. Class. Quantum Gravity 20, S473 (2003)

7. D. Francia, A. Sagnotti, On the geometry of higher spin gauge fields. Comment. Phys. Math. Soc. Sci. Fenn. 166, 165 (2004)

8. D. Francia, A. Sagnotti, On the geometry of higher spin gauge fields. PoS JHW 2003, 005 (2003). arXiv:hep-th/0212185

9. D. Francia, A. Sagnotti, Free geometric equations for higher spins. Phys. Lett. B 543, 303 (2002). arXiv:hep-th/0207002

10. F.A. Berends, G.J.H. Burgers, H. Van Dam, On the theoretical problems in constructing interactions involving higher-spin massless particles. Nucl. Phys. B 260, 295-322 (1985)

11. C. Fronsdal, Massless fields with integer spin. Phys. Rev. D 18, 3624 (1978)

12. J. Fang, C. Fronsdal, Massless fields with half integral spin. Phys. Rev. D 18, 3630 (1978)

13. X. Bekaert, E. Joung, J. Mourad, On higher spin interactions with matter. JHEP 0905, 126 (2009). arXiv:0903.3338 [hep-th]

14. X. Bekaert, E. Joung, J. Mourad, Effective action in a higher-spin background. JHEP 1102, 048 (2011). arXiv:1012.2103 [hep-th]

15. L. Bonora, M. Cvitan, P. Dominis Prester, S. Giaccari, M Paulišić, T. Štemberga, Worldline quantization of field theory, effective actions and $L_{\infty}$ structure. JHEP 1804, 095 (2018). arXiv: 1802.02968 [hep-th]

16. O. Hohm, B. Zwiebach, $L_{\infty}$ algebras and field theory. Fortschr. Phys. 65(3-4), 1700014 (2017). arXiv:1701.08824 [hep-th]

17. M.R. Gaberdiel, B. Zwiebach, Tensor constructions of open string theories. 1: Foundations. Nucl. Phys. B 505, 569 (1997). arXiv:hep-th/9705038

18. B. Zwiebach, Oriented open-closed string theory revisited. Ann. Phys. 267, 193 (1998). arXiv:hep-th/9705241

19. H. Kajiura, J. Stasheff, Homotopy algebras inspired by classical open-closed string field theory. Commun. Math. Phys. 263, 553 (2006). arXiv:math/0410291 [math-qa]

20. T. Lada, J. Stasheff, Introduction to SH Lie algebras for physicists. Int. J. Theor. Phys. 32, 1087 (1993). arXiv:hep-th/9209099

21. T. Lada, M. Markl, Strongly homotopy Lie algebras. Commun. Algebra 23, 2147 (1995). arXiv:hep-th/9406095 
22. G. Barnich, R. Fulp, T. Lada, J. Stasheff, The SH Lie structure of Poisson brackets in field theory. Commun. Math. Phys. 191, 585 (1998). arXiv:hep-th/9702176

23. A.M. Zeitlin, String field theory-inspired algebraic structures in gauge theories. J. Math. Phys. 50, 063501 (2009). arXiv:0711.3843 [hep-th]

24. A.M. Zeitlin, Conformal field theory and algebraic structure of gauge theory. JHEP 1003, 056 (2010). arXiv:0812.1840 [hep-th]

25. T. Lada, $L_{\infty}$ algebra representations. Appl. Categ. Struct. 12, 29$34(2004)$

26. J. Stasheff, Unpublished

27. R. Fulp, T. Lada, J. Stasheff, Sh-Lie algebras induced by gauge transformations. Commun. Math. Phys. 231, 25 (2002)

28. A.D. Sakharov, Vacuum quantum fluctuations in curved space and the theory of gravitation. Sov. Phys. Dokl. 12, 1040 (1968)

29. A.D. Sakharov, Vacuum quantum fluctuations in curved space and the theory of gravitation. Dokl. Akad. Nauk Ser. Fiz. 177, 70 (1967)

30. A.D. Sakharov, Vacuum quantum fluctuations in curved space and the theory of gravitation. Sov. Phys. Usp. 34, 394 (1991)

31. A.D. Sakharov, Vacuum quantum fluctuations in curved space and the theory of gravitation. Gen. Relativ. Gravit. 32, 365 (2000)

32. L. Bonora, M. Cvitan, P. Dominis Prester, B. Lima de Souza, I. Smolić, Massive fermion model in 3d and higher spin currents. JHEP 1605, 072 (2016). arXiv:1602.07178 [hep-th]

33. L. Bonora, M. Cvitan, P. Dominis Prester, S. Giaccari, B. Lima de Souza, T. Štemberga, One-loop effective actions and higher spins. JHEP 1612, 084 (2016). arXiv:1609.02088 [hep-th]

34. L. Bonora, M. Cvitan, P. Dominis Prester, S. Giaccari, T. Štemberga, One-loop effective actions and higher spins. II. JHEP 1801, 080 (2018). arXiv:1709.01738 [hep-th]

35. M.J. Strassler, Field theory without Feynman diagrams: one loop effective actions. Nucl. Phys. B 385, 145 (1992). arXiv:hep-ph/9205205
36. A.Y. Segal, Conformal higher spin theory. Nucl. Phys. B 664, 59 (2003). arXiv:hep-th/0207212

37. A.Y. Segal, Point particle in general background fields versus gauge theories of traceless symmetric tensors. Int. J. Mod. Phys. A 18, 4999 (2003). arXiv:hep-th/0110056

38. M.G. Schmidt, C. Schubert, The Worldline path integral approach to Feynman graphs. In Ahrenshoop Symposium (1994), pp. 240253. arXiv:hep-ph/9412358

39. M.G. Schmidt, C. Schubert, Worldline Green functions for multiloop diagrams. Phys. Lett. B 331, 69 (1994). arXiv:hep-th/9403158

40. P. Dai, W. Siegel, Worldline Green functions for arbitrary Feynman diagrams. Nucl. Phys. B 770, 107 (2007). arXiv:hep-th/0608062

41. R. Bonezzi, Induced action for conformal higher spins from worldline path integrals. Universe 3(3), 64 (2017). arXiv:1709.00850 [hep-th]

42. L. Bonora, M. Cvitan, P. Dominis Prester, S. Giaccari, T. Štemberga, HS in flat spacetime. YM-like models. arXiv:1812.05030 [hep-th]

43. V.E. Lopatin, M.A. Vasiliev, Free massless bosonic fields of arbitrary spin in $d$-dimensional De Sitter space. Mod. Phys. Lett. A 3, 257 (1988). https://doi.org/10.1142/S0217732388000313

44. E.E. Boos, A.I. Davydychev, A method of evaluating massive Feynman integrals. Theor. Math. Phys. 89, 1052 (1991)

45. E.E. Boos, A.I. Davydychev, A method of evaluating massive Feynman integrals. Teor. Mat. Fiz. 89, 56 (1991)

46. A.I. Davydychev, A simple formula for reducing Feynman diagrams to scalar integrals. Phys. Lett. B 263, 107 (1991)

47. A.I. Davydychev, Recursive algorithm of evaluating vertex type Feynman integrals. J. Phys. A 25, 5587 (1992) 